\title{
Recent progress in flexible energy storage materials for lithium-ion batteries and electrochemical capacitors: A review
}

\author{
Zhiyu Wang, Weike Zhang, Xuelian Li, and Lizhen Gao ${ }^{\text {a) }}$ \\ College of Environmental Science and Engineering, Taiyuan University of Technology, Taiyuan 030024, China
}

(Received 15 March 2016; accepted 25 April 2016)

\begin{abstract}
With the advent of flexible, wearable and portable electronic products, flexible lithium-ion batteries (LIBs) and electrochemical capacitors (ECs), which are able to withstand repeated deformation or bending, have attracted considerable attention as one type of energy-storage device. However, the fabrication of these flexible electrodes is the main bottleneck in the practical utilization and application of these energy-storage devices. Up to now, enormous efforts have been made in addressing the shortcomings and remarkable improvements have also been achieved. So a systematic review of the status and progresses is highly required. In this review, we first make a short introduction about the challenges faced in the conventional batteries and capacitors. Then, we summarize the recent improvements in flexible and wearable LIBs and ECs with a focus on the flexible active materials and substrates. Finally, we discuss the prospects and challenges towards the practical applications of the flexible electrodes in the future.
\end{abstract}

\section{INTRODUCTION}

In recent years, flexible or bendable energy storage and conversion systems, which are designed to be portable, lightweight, bendable and even wearable, have attracted tremendous attention due to their mechanical flexibility and high energy density. ${ }^{1-3}$ Among various energy-storage devices, lithium-ion batteries (LIBs) and electrochemical capacitors (ECs) are two of the most promising candidates because of their high energy density, large power density and excellent stability. ${ }^{4}$ Due to the excellent properties of flexible devices, LIBs and ECs, a combination of flexible electrodes with LIBs and ECs has attracted much more interest as an important branch of bendable electronic systems.

However, the bottlenecks of transforming the traditional batteries and capacitors into flexible energy storage devices lie in the preparation methods, assembly process, and the selection of proper electrolytes. Conventional LIBs and ECs usually consist of a carbon-based anode, a transition metal oxides-based cathode, a polymer separator and organic liquid electrolyte. However, there are several disadvantages for the traditional methods. In these batteries, relatively heavy metal foils are always used as both conductive substrate and structural support with slurry of active materials, binders and conductive additives coated on the surface of it. Due to the smooth surface of the metal foil, the active materials can easily detach

Contributing Editor: Xiaobo Chen

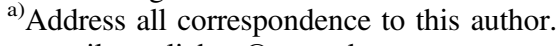

e-mail: gaolizhen@tyut.edu.cn

DOI: $10.1557 / j m r .2016 .195$ from the current collector. In addition, the relatively poor chemical stability of these metal foils also results in increased internal impedance ${ }^{5}$ and passivation of active materials, ${ }^{6}$ because metal foils are susceptible to the corrosion of some electrodes and active materials. Carbon black, carbon nanotubes (CNTs) and graphite are always used as conductive additives, which account for about $10-20 \mathrm{wt} \%$ of the whole slurry, to improve the conductivity of the whole electrode. Nevertheless, they have no or little contribution to the capacity of the electrodes. Some side effects are also caused by the polymer binders such as polyvinylidene fluoride (PVDF), polytetrafluoroethylene, which are electrochemically inactive or even insulating. So the existence of these binders further hinders the transport of lithium-ion (Li-ions) and electrons within the active materials and compromises the overall energy density of the electrodes.

Compared with the metal foil-based electrodes, flexible and binder-free electrodes have their unique advantages in terms of portability, energy density and cycling stability over the conventional electrodes. Most of the flexible electrodes replace the traditional binders and heavy current collectors with flexible and light substrates or inbedded current collectors. Up to now, there are mainly two approaches to make a combination of the active materials and the flexible substrates. The first one is to utilize the conductive and flexible matrix as embedded in current collector or substrates with active materials firmly entrapped on them through coating, spraying, deposing or other novel methods. The second method is adopting a nonconductive substrate with excellent mechanical properties such as polydimethylsiloxane (PDMS), plastic and 
cotton as substrates. Despite these novel approaches, there are still several challenges for flexible and portable energy-storage devices.

(1) Among the traditional active materials used as anodes or cathodes for LIBs and ECs, most of them are intrinsically inflexible materials such as transition metal oxides, sulfur, silicon and so on. To further apply these active materials in flexible electrodes, a mechanically robust substrate, conductive or nonconductive, must be adopted in the whole system.

(2) Liquid electrolytes are still the dominant electrolytes used in the whole flexible systems due to their excellent electrical conductivity. However, apart from the possible safety concerns like electrolyte leakage and toxicity, liquid electrolytes also limit the design and assembly of the cells, thus hindering their practical applications. Solid-state electrolytes with excellent mechanical strength and robustness hold great potential in solving the above mentioned shortages, but the development of this kind of electrolytes is still at an early stage and cannot meet the demand for flexible electronic devices.

(3) The mass loading of the active materials on the flexible materials is always compromised to achieve a high level of flexibility and mechanical strength, which is of practical importance in their applications in the portable device market.

The review is aim to summarize the recent progress in flexible LIBs and ECs, with a focus on flexible active materials and substrates, and discuss the possible challenges and prospects in the near future.

\section{CNT BASED FLEXIBLE ELECTRODES}

CNTs can be envisioned as rolled up graphene sheets and used as Li-ion storage materials, which can be attributed to the diffusion of Li-ion on the surface and between the layers of CNTs. In recent years, as a successfully commercialized product with high electrical conductivity, high flexibility, high Young's modulus and exceptional surface properties, CNTs have attracted enormous attention and are widely used as active materials in flexible LIBs and supercapacitors as high performance anode materials. It has been reported in the literature that it is possible to charge single-wall carbon nanotubes (SWCNTs) up to $1000 \mathrm{~mA} \mathrm{~h} / \mathrm{g}$ for the first cycle, ${ }^{7,8}$ which results from effective diffusion of Li-ions on the surface and/or inside individual nanotubes. ${ }^{9}$ However, the anode exhibits an irreversible capacity during the initial cycle, which may be caused by the defects in the CNTs and the formation solid-electrolyte-interface (SEI). ${ }^{10}$ In terms of electrochemical performance, multi-wall CNTs (MWCNT) exhibit better reversible charge and discharge storage capacity than SWCNTs and double-wall CNTs. ${ }^{11}$ Apart from different types of CNTs, it is well documented that the preparation methods, ${ }^{10}$ the electrolytes, ${ }^{12}$ the surface properties ${ }^{13}$ and elemental doping of $\mathrm{CNT}^{14}$ also greatly influence the capacity of CNT as Li-ion storage materials. For example, Lee's group ${ }^{15}$ reported that the redox of oxygen-containing functional groups on the surface of CNT such as MWNT-COOH and MWNT$\mathrm{NH}_{2}$ with Li-ions is responsible for the large gravimetric capacitances of MWNT electrodes in organic electrolytes.

Another advantage of CNT is that it can eliminate the use of conventional $\mathrm{Cu}$ or Al-based current collectors, conductive additives and binders and serve as flexible and porous current collectors. Therefore, various forms of CNT-based flexible materials such as yarns, films and fibers have been reported as feasible materials for broad applications. ${ }^{16}$ Meanwhile, some novel methods such as spraying, ${ }^{17}$ dip-coating, ${ }^{18}$ vacuum filtration ${ }^{19}$ have also been explored to fabricate such flexible and highperformance electrodes with CNT as active materials or current collectors.

Among the recently developed methods, vacuum filtration has been demonstrated to be the most commonly used approach to fabricate CNT and active materials composite film with interconnected porous channels for fast ion and electron transport. For example, $\mathrm{MnO}_{2} / \mathrm{CNT} /$ conductive polymer (CP) film with good cycling and rate stability was synthesized by Hou et al. ${ }^{20}$ Acid treatment was used to add functional groups such as carboxylic groups or hydroxyl groups on the surface of CNTs, since the polar functional groups are able to effectively adsorb the $\mathrm{Mn}^{2+}$ ions in the solution, ensuring intimate contact between CNT and $\mathrm{MnO}_{2}$, as shown in Fig. 1. The film with a high mass loading of $\mathrm{MnO}_{2}(60 \%)$ delivers specific capacitance value as high as $200 \mathrm{~F} / \mathrm{g}$, which demonstrates a high utilization of the active materials with assistance of the poly(3,4-ethylenedioxythiophene)-poly(styrenesulfonic acid) (PEDOT-PSS) and the functional groups of the CNTs.

Nevertheless, directly mixing the active materials and CNT via simple dispersion and filtration may yield a weak interaction between the conductive nets and active materials, resulting in a relatively poor electrochemical performance of the flexible electrodes. To address the problem, a two-step in situ method was applied by Jia et al. to synthesize $\mathrm{LiMn}_{2} \mathrm{O}_{4}$ based CNT films. ${ }^{21}$ Layers of $\mathrm{MnO}_{2}$ were firstly generated on the surface of CNT by redox reactions between the CNTs and $\mathrm{KMnO}_{4}$. Then a hydrothermal reaction further converted the $\mathrm{MnO}_{2}$ into $\mathrm{LiMn}_{2} \mathrm{O}_{4}$ particles. Subsequent vacuum filtration of the $\mathrm{LiMn}_{2} \mathrm{O}_{4} / \mathrm{CNT}$ composites generated a self-standing and binder-free film. At a current density of $550 \mathrm{~mA} \mathrm{~h} / \mathrm{g}$, the composite material delivers a discharge capacity of $50 \mathrm{~mA} \mathrm{~h} / \mathrm{g}$. To further improve the interaction between the active materials and the conductive scaffolds, a novel strategy was reported by Cheng et al. ${ }^{22}$ They mixed $\mathrm{TiO}_{2}$ and CNT in water in the existence of sodium lauryl benzenesulfate (SDBS) to form a black suspension, as shown in Fig. 2. Then the black suspension was filtered 


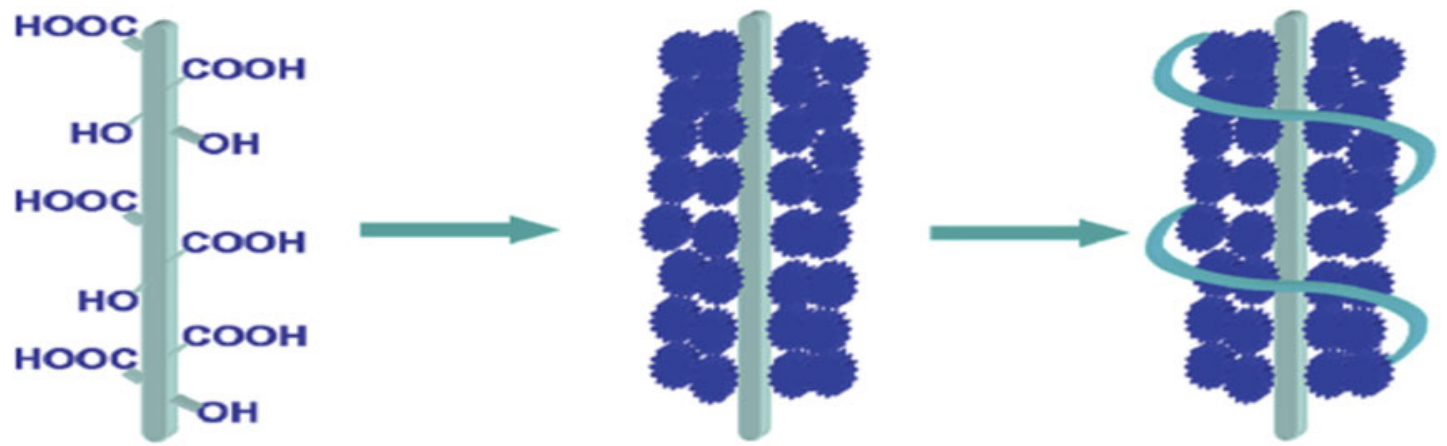

(a)

Carbon nanotubes

$\mathrm{MnO}_{2}$ nanoparticles

PEDOT-PSS
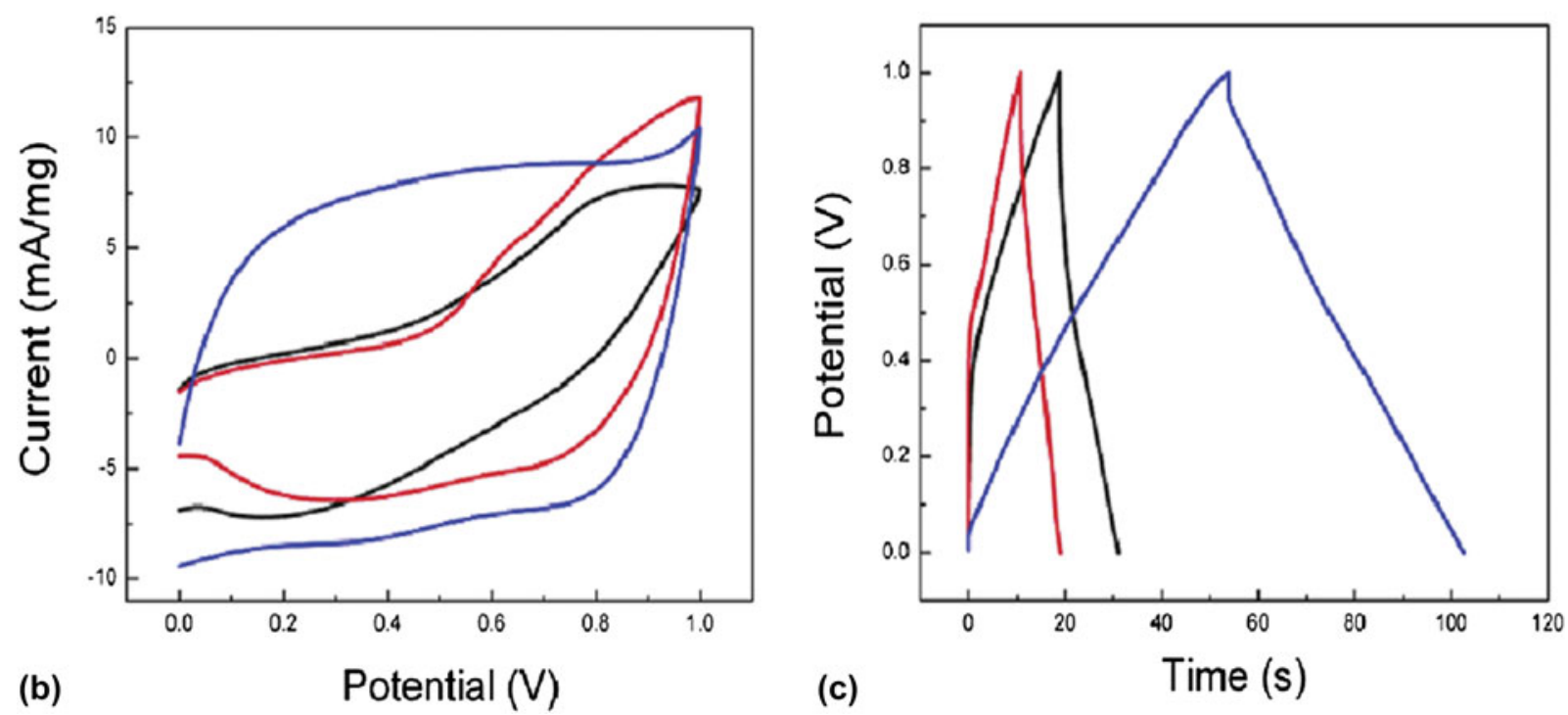

FIG. 1. (a) A schematic illustration of $\mathrm{MnO}_{2} / \mathrm{CNTs} / \mathrm{PEDOT}-\mathrm{PSS}$ composites. (b) Cyclic voltammograms (50 mV/s) and (c) galvanostatic charge/ discharge curve (at current density of $5 \mathrm{~mA} / \mathrm{cm}$ ) of $\mathrm{MnO}_{2}$ film (black), $\mathrm{MnO}_{2} / \mathrm{PEDOT}-\mathrm{PSS}$ composite (red), and $\mathrm{MnO} / 2 \mathrm{CNT} / \mathrm{PEDOT}$-PSS composite (blue). Reprinted with permission from Ref. 20, Copyright 2010 American Chemical Society.

by a porous membrane to form a film. A hydrothermal reaction in $\mathrm{NaOH}$ aqueous solution and a heating treatment were used to improve the adhesion and interaction between active materials and the conductive scaffolds by conformal coating $\mathrm{TiO}_{2}$ nanorods on the surface of CNTs. The results show that a charge capacity of $129 \mathrm{~mA} \mathrm{~h} / \mathrm{g}$ is maintained after 1000 cycles at $10 \mathrm{C}$.

However, the disadvantages of the acid treatment and surfactants lie in the reduction of the conductivity of the CNT, which is caused by the sluggish kinetics of the functional groups and surfactants on the surface of CNTs. ${ }^{23}$ Depositing silicon coatings onto CNT has been proven by Fu et al. as an effective way to utilize the remarkable properties of both CNT and silicon. ${ }^{24}$ CNT forests were grown on a quartz substrate with $\mathrm{SiH}_{4}$ gas used as the silicon source for the uniform deposition of silicon layers. The aligned CNT provides sufficient inter-tube space for the uniform deposition of silicon coatings and accommodates the silicon's volume expansion during cycling. The cycling test shows that the charge capacity is $1494 \mathrm{~mA} \mathrm{~h} / \mathrm{g}$ after 45 cycles with a capacity retention of over $94 \%$.

$\mathrm{CP}$ solution can also be used as optimal "conductive surfactants" to replace the conventional surfactants or acid treatment for the preparation of free-standing and binder CNT film. Chen et al. used $\mathrm{TiO}_{2}$ nanoparticles, PEDOT:PSS solution and superlong CNTs to make a hydrogel precursor (see Fig. 3). ${ }^{25}$ PEDOT:PSS was used as both conductive additives and surfactants since it contains both hydrophobic part and hydrophilic part. A flat film was also formed by vacuum filtration. The CNT/CP 


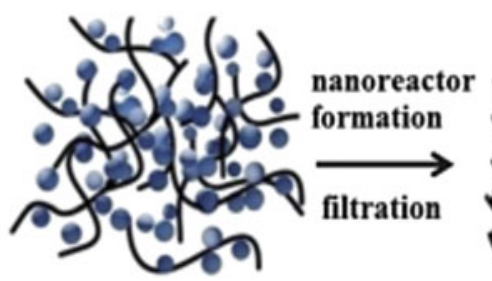

CNTs and $\mathrm{TiO}_{2}$ particles dispersion

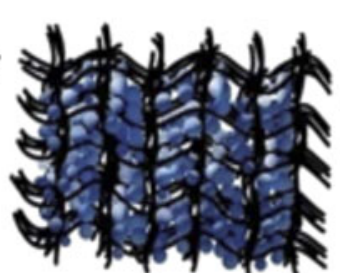

$\mathrm{CNT} / \mathrm{TiO}_{2}$ mixed film conformal coating

confined reaction

Conformal CNT-TiO, film

(a)
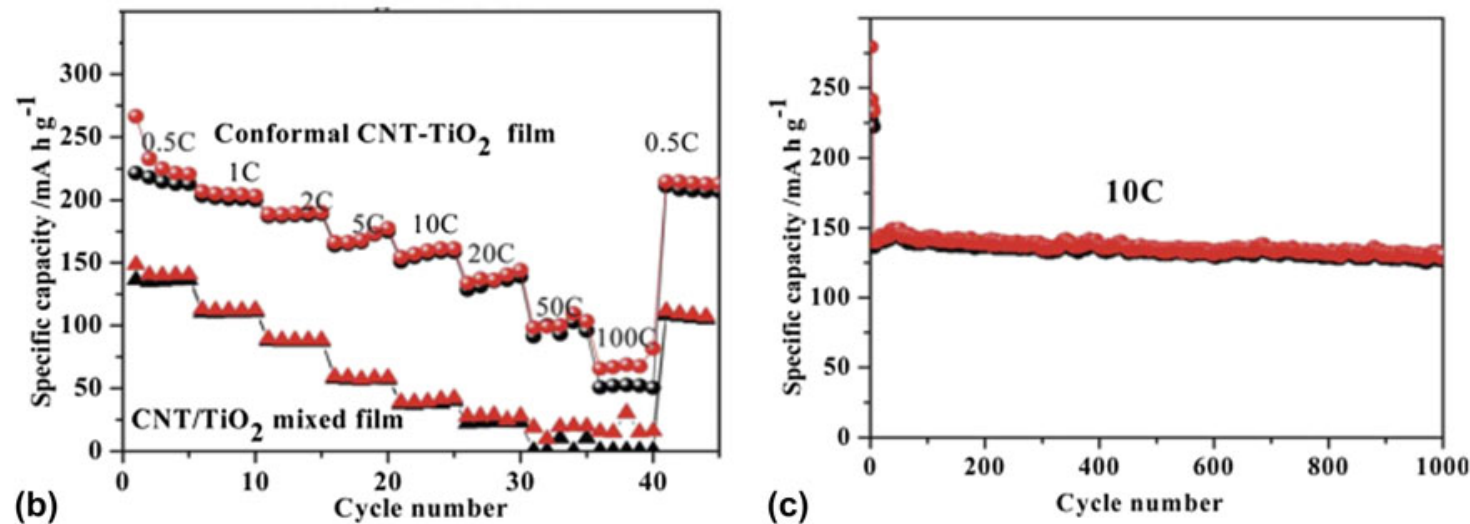

FIG. 2. (a) Schematic of preparing a free-standing and binder-free $\mathrm{CNT}-\mathrm{TiO}_{2}$ film by conformal coating of $\mathrm{TiO}_{2}$ on a 3-D CNT scaffold. (b) The rate capability of the conformal $\mathrm{CNT}-\mathrm{TiO}_{2}$ film and $\mathrm{CNT}-\mathrm{TiO}_{2}$ mixed electrodes. (c) Cycling performance of the conformal $\mathrm{CNT}-\mathrm{TiO}{ }_{2}$ film electrode at a high current rate of 10 C. Reprinted with permission from Ref. 22, Copyright 2014 Royal Society of Chemistry.
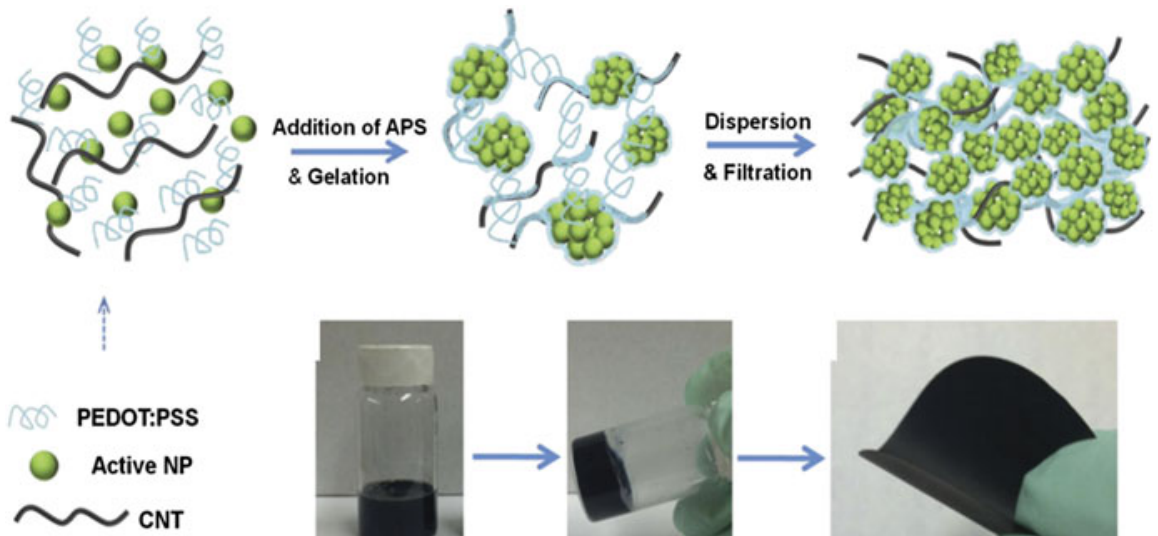

(a)

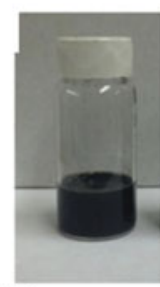

(b)

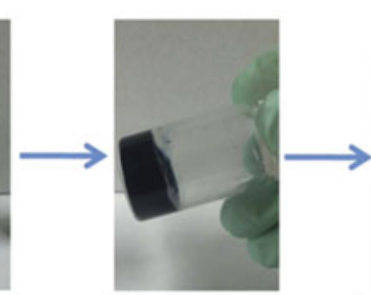

(c)

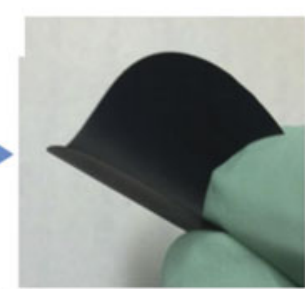

(d)

FIG. 3. (a) Schematic of the aqueous solution process to fabricate flexible electrodes. (b) Photograph of the precursor solution. (c) Photograph of a composite hydrogel. (d) Photograph of an example of a flexible $\mathrm{TiO}_{2}-$ PEDOT:PSS-CNT film. Reprinted with permission from Ref. 25, Copyright 2014 WILEY-VCH Verlag GmbH \& Co. KGaA, Weinheim.

film functions as an interpenetrating network which facilitates electrode kinetics and improves strain tolerance.

CNTs can also been spun into continuous fibers with desirable diameters and lengths through wet spinning processes $^{26}$ and chemical vapor deposition (CVD). ${ }^{27}$ The advantages of these CNT-based fiber such as low density and high electrical conductivity make it attractive to be integrated with various active materials to achieve flexible and high performance electrodes.

In this respect, Lin et al. reported a CNT/silicon composite fiber anode for flexible wire-shaped LIBs. ${ }^{28}$ Aligned MWCNT arrays were synthesized by CVD followed by deposition of Si through electron beam evaporation to form a composite film. The film was further 
twisted into fibers and used as working electrodes with lithium wire as counter electrode. The MWCNT/Si composite fiber exhibits a specific capacity of $1042 \mathrm{~mA} \mathrm{~h} / \mathrm{g}$ at $3.0 \mathrm{~A} / \mathrm{g}$ after 10 cycles at $2.0 \mathrm{~A} / \mathrm{g}$ and 10 cycles at $3.0 \mathrm{~A} / \mathrm{g}$. The good electrochemical performance of the CNT/Si composite fiber can be attributed to the core-sheath structure, which effectively accommodate silicon's volume changes.

\section{GRAPHENE BASED FLEXIBLE ELECTRODES}

The unique nanostructure of graphene with twodimensional (2-D) carbon layers has drawn tremendous attention because it possesses numerous appealing properties such as high surface area, excellent conductivity, low weight, excellent chemical stability and high mechanical flexibility and strength. ${ }^{29-31}$ Since the breakthrough made in the experimental discovery of individual graphene in $2004,{ }^{32}$ tremendous attempts have been made to produce individual graphene nanosheets such as $\mathrm{CVD},{ }^{33-35}$ chemical modification ${ }^{36}$ and chemical exfoliation. ${ }^{37}$

Similarly, graphene can be used in both LIBs and ECs as Li-ion host materials. The electrochemical capacitance of graphene can be divided into two categories: electrochemical double-layer capacitors and pseudocapacitors. The former capacitance can be achieved through an adsorption and intercalation mechanism. ${ }^{38}$ The pseudocapacitance results from some electroactive species such as residual oxygen-containing and hydrogen-containing functional groups on the surface of graphene after various chemical modification of graphene. ${ }^{39}$ However, it is of extraordinary challenge to directly assemble individual 2-D graphene sheet into controllable graphene-based nanoreactor or nanosystem due to its hydrophobicity, aggregation and restacking. ${ }^{40}$ Therefore, much effort has been devoted to resolve this drawback.

As one type of graphene sheets assembled microcosmic carbon materials, graphene films are the most commonly used active materials or current collectors in flexible LIBs and ECs. In this respect, $\mathrm{Li}$ et al. did some pioneering works in the preparation of macroscopic scale graphene papers. ${ }^{41}$ They reported that stable aqueous colloids of chemically converted graphene (CCG) can be obtained without the need for surfactant or stabilizers. Uniform graphene films can be formed on a membrane filter by vacuum filtration of the CCG dispersions. Later, it has been demonstrated in the same group that the mechanical robustness and strength of as-synthesized CCG film can be enhanced by moderate thermal annealing. ${ }^{42}$ To further fulfil its potential in LIBs and ECs, vacuum filtration of CCG and polyaniline nanofibers (PANI-NFs) was reported by $\mathrm{Wu}$ et al. to form a composite film with PANI-NFs sandwiched between CCG layers. ${ }^{43}$ The flexible supercapacitor devices based on the film show electrochemical capacitance of $210 \mathrm{~F} / \mathrm{g}$ at a discharge rate of $0.3 \mathrm{~A} / \mathrm{g}$.
Yang et al. also demonstrated a liquid-mediated method by which multilayered graphene sheets can remain largely separated due to the intrinsic corrugation and colloidal interaction between as-synthesized CCG sheets. ${ }^{44}$ Apart from the filtration-based methods, a facile method has also been reported by Shen et al. to fabricate large graphene dioxide (GO) sheets film by direct evaporation of GO suspension under mild heating. After graphitization at a high temperature of $2000{ }^{\circ} \mathrm{C}$ for $1 \mathrm{~h}$ under argon flow, the graphene film shows a graphite-like structure, excellent electromagnetic interface and in-plane thermal conductivity. ${ }^{45}$

At the same time, great efforts have been made to further improve the strength and conductivity of graphenebased flexible film, fiber or other forms of materials by enhancing the interlayer interactions between graphene sheets. ${ }^{46}$ Multifunctional polymers such as multi-amino polyallylamine, have been applied to covalently crosslink graphene sheets due to the reaction between amine groups and the functional groups of the GO, but severe aggregation of GO sheets cannot be avoided to some extent. ${ }^{47}$ Later, a controllable crosslinking method was reported by Tian et al. using polydopamine as cross-linking agents, which have the capacity to adhere to various substrates. ${ }^{48}$ When the $\mathrm{pH}$ value of the system is above 8.5 , the musselinspired polymers are able to react with amine groups. ${ }^{49}$ By tuning the $\mathrm{pH}$ of the solution, a controllable crosslink reaction can be realized.

However, the reversible capacity of the graphene film is still hindered by aggregation and restacking of individual graphene sheets, which inhibit the infiltration of electrolytes and the diffusion of Li-ion in both LIBs and ECs to some extent. Recently, interconnected and porous graphene materials have aroused great interest because it has been demonstrated that porous graphene structures with large surface area and interconnected nanopores are helpful in enhancing the accessibility of electrolyte and improve capacitance characteristics. ${ }^{50-52}$ Chen et al. reported three-dimensional graphene foam (GF) synthesized by template-directed CVD. ${ }^{53}$ Carbon was introduced into a nickel foam by decomposing $\mathrm{CH}_{4}$ at $1000{ }^{\circ} \mathrm{C}$ on the surface of the nickel foam. The interconnected and flexible network of graphene with large surface area provides fast transport channel for Li-ion and electrons. Based on this concept, the free-standing and porous GF network was fabricated and used as current collector for lithium-sulfur batteries $(\mathrm{Li}-\mathrm{S})$ by $\mathrm{Xi}$ et al. ${ }^{54}$ After infiltrating the sulfur solution into GF by a drop casting method, the binder-free and flexible $\mathrm{Li}-\mathrm{S}$ electrodes were prepared and shows excellent rate and cycling ability with a Coulombic efficiency of $94.6 \%$ after 400 cycles at a high current density.

As mentioned previously, the porous structure of GF is necessary in improving the electrochemical performance of the GF. To further utilize the porous structure, GF with uniform and controllable pore size distribution was 
reported by Huang et al. ${ }^{55}$ They designed a hydrophobic interaction driven hard templating approach to prepare GF with controllable pore size $(30-120 \mathrm{~nm})$ and high pore volumes (around $4.3 \mathrm{~cm}^{3} / \mathrm{g}$ ). Silica spheres were chosen for the preparation of GF followed by etching with HF. Due to the hydrophobic and porous surface nature of the GF, metal oxide nanoparticles can be easily entrapped on the surface of the walls of GF. A typical NGF with a uniform particle size of $27.7 \mathrm{~nm}$ is able to deliver a large reversible capacity of about $750 \mathrm{~mA} \mathrm{~h} / \mathrm{g}$ at $300 \mathrm{~mA} / \mathrm{g}$.

The methods discussed above require a high temperature or complex multi-step processes like CVD or etching. As shown in Fig. 4, a novel and simple strategy was reported by Liu et al. to make graphene paper with 3-D porous network by mechanically pressing a graphene aerogel, which was synthesized by freeze-drying a GO aqueous dispersion followed by thermal reduction. ${ }^{56}$ The folded structure of the graphene sheets with fewer layers can provide more active sites for hosting Li-ion. The electrochemical capacity is up to $172 \mathrm{~F} / \mathrm{g}$ and $110 \mathrm{~F} / \mathrm{g}$ at a charge/discharge rate of $1 \mathrm{~A} / \mathrm{g}$ and $100 \mathrm{~A} / \mathrm{g}$, respectively, which is higher than that of graphene paper fabricated by the conventional flow-directed assembly methods. ${ }^{57}$

When integrated with other active materials such as $\mathrm{V}_{2} \mathrm{O}_{5},{ }^{58} \mathrm{MnO}_{2},{ }^{59} \mathrm{TiO}_{2},{ }^{60}$ silicon, ${ }^{61}$ sulfur ${ }^{62}$ and other active materials to form a flexible electrode, GFs and films exhibit their unique properties including extremely high specific surface area, excellent electronic conductivity and chemical stability. Moreover, the main advantage of the 2-D layer structure of graphene lies in the formation of mechanically robust matrix, the stronger adhesion between the active materials and graphene, the uniform distribution of the active materials and the elimination of the metal current collectors, conductive additives and binders.

Magnetite $\left(\mathrm{Fe}_{3} \mathrm{O}_{4}\right)$ is regarded as a very appealing active material for LIBs due to its high theoretical capacity $(926 \mathrm{~mA} \mathrm{~h} / \mathrm{g})$ and environmental benignity. ${ }^{63} \mathrm{~A}$ combination of graphene film with $\mathrm{Fe}_{3} \mathrm{O}_{4}$ has been reported by Wang et al. to prepare a free-standing and flexible $\mathrm{Fe}_{3} \mathrm{O}_{4} /$ graphene hybrid film. ${ }^{64}$ Graphene sheets functions as a 3-D conductive matrix with $\mathrm{Fe}_{3} \mathrm{O}_{4}$ homogeneously distributed and firmly entrapped in its porous and rough surface. $\mathrm{Fe}_{2} \mathrm{O}_{3}$ was used as precursor and mixed with GO to form a film via filtration. Then the film was heated at $600{ }^{\circ} \mathrm{C}$ in argon flow for $1 \mathrm{~h}$ for chemical conversion to fabricate $\mathrm{Fe}_{3} \mathrm{O}_{4} /$ graphene film. The as synthesized film graphene exhibits a high specific capacity of 1555 , 940 and $660 \mathrm{~mA} \mathrm{~h} / \mathrm{g}$ at 100, 200 and $500 \mathrm{~mA} / \mathrm{g}$. Similarly, filtration was also used to prepare $\mathrm{Co}_{3} \mathrm{O}_{4} /$ graphene hybrid film. ${ }^{65}$ Based on the total electrode weight, the hybrid film delivers a high specific capacity of 1400 and $1200 \mathrm{~mA} \mathrm{~h} / \mathrm{g}$ at 100 and $200 \mathrm{~mA} / \mathrm{g}$, respectively.

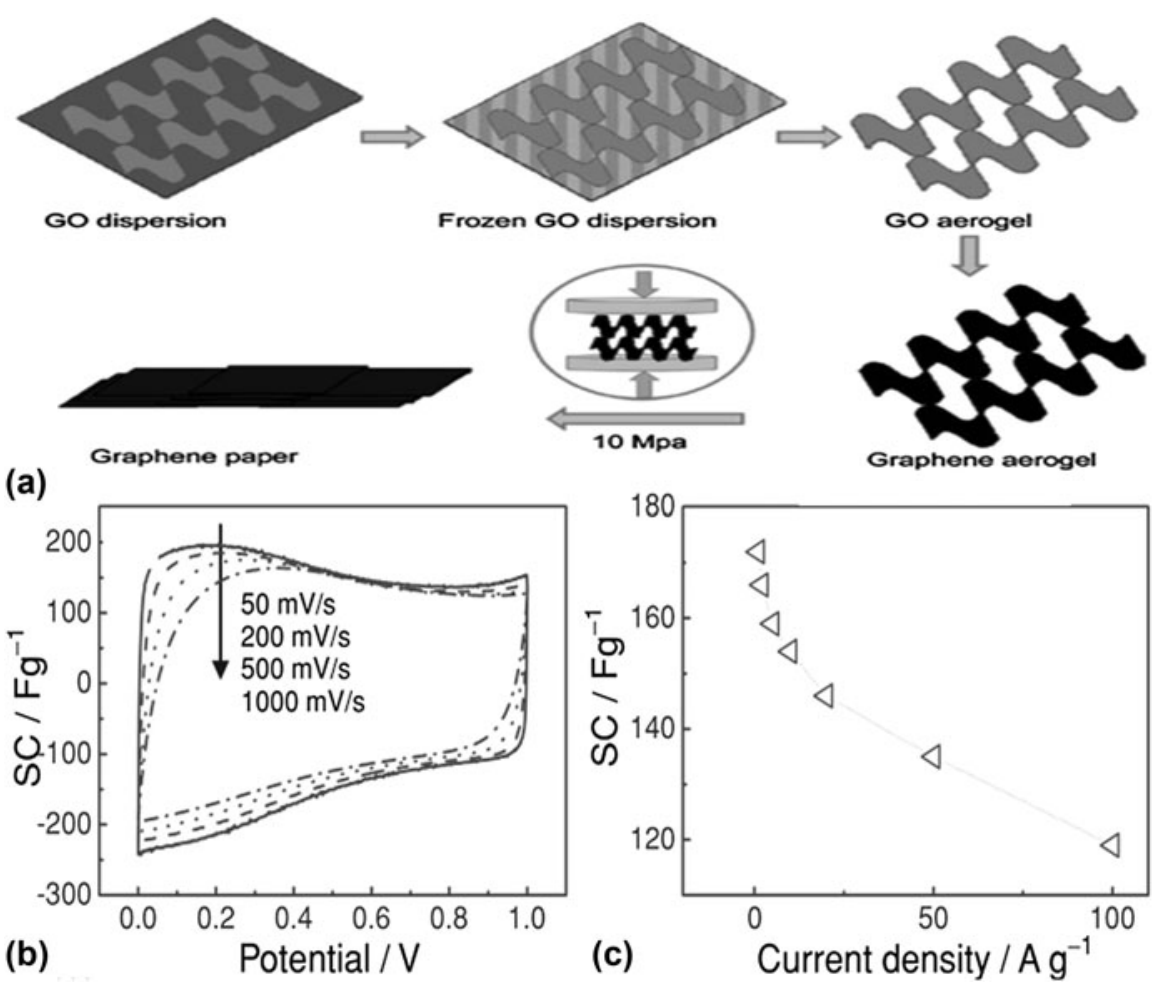

FIG. 4. (a) Illustration of the formation process of graphene paper. (b) CV curves of the graphene paper as a supercapacitor electrode at different scan rates. (c) Specific capacitance of the graphene paper as a function of charge/discharge rate. Reprinted with permission from Ref. 56, Copyright 2012 WILEY-VCH Verlag GmbH \& Co. KGaA, Weinheim. 
Apart from the graphene films, ultrathin graphite foam (UGF) loaded with lithium iron phosphate (LFP) was used as a cathode for LIB by Ji et al. ${ }^{66}$ LFP was mixed with carbon black and PVDF at a weight ratio of 7:2:1 in $\mathrm{N}$-methyl-2-pyrrolidone to form slurry, which was dropcast on the UGF to produce a cathode. A higher rate capability and specific capacity can be achieved (23\% higher than that of the Al/LFP cathode and 170\% higher than that of the Ni-foam/LFP cathode) simultaneously, owing to the conductive and 3-D interconnected graphitic structure.

In addition to the progresses achieved in various graphene papers and foams, one dimensional (1-D) graphene-based fibers are of practical importance in constructing high-strength, portable and high energy density devices. Various methods such as CVD, ${ }^{67}$ electrophoretic assembly method ${ }^{68}$ and facile solution self-assembly strategy, ${ }^{69}$ have been made in the fabrication of graphene fibers.

Wet spinning has been demonstrated as a breakthrough to fabricate graphene fibers in recent years. ${ }^{70,71}$ Various coagulation baths with different solvents such as methyl acetate, ethyl acetate and $\mathrm{CaCl}_{2}$ have been used to improve the mechanical stiffness and morphology of the graphene fibers. ${ }^{72}$ Aboutalebi et al. reported a multifunctional graphene yarns by taking advantage of the intrinsic self-assembly behavior of ultra large graphene oxide liquid dispersions. ${ }^{73}$ Ultra large reduced GO (RGO) sheets with above $50 \mu \mathrm{m}$ large sheet in size are demonstrated of great importance in achieving both high conductivity and electrochemical capacitance because of its uninterrupted surface and less grain boundaries (Fig. 5). A similar experiment conducted by Xiang et al. confirmed that larger building blocks usually result in carbon graphene-based fibers with better mechanical properties. $^{74}$ In their experiment, ethyl acetate was selected as the coagulation solvent for the experiment followed by a heat treatment at $1000{ }^{\circ} \mathrm{C}$. The continuous fibers yield a modulus of $47 \mathrm{GPa}$, which was much higher than conventional pure graphene-based fibers.

In addition to the conventional methods, a novel facile one-step dimensionally confined hydrothermal strategy was reported by Qu's group to prepare a neat graphene fiber from GO suspensions. ${ }^{75}$ A glass pipeline was used as the reactor and aqueous GO suspension was injected into the glass pipeline, followed by low-temperature heat treatment at $230{ }^{\circ} \mathrm{C}$ for $2 \mathrm{~h}$. A strong and low-weight graphene fiber well matching the pipe geometry was produced. Later, to extend the applications of as-synthesized fibers, $\mathrm{MnO}_{2}$-modified graphene fiber has been fabricated
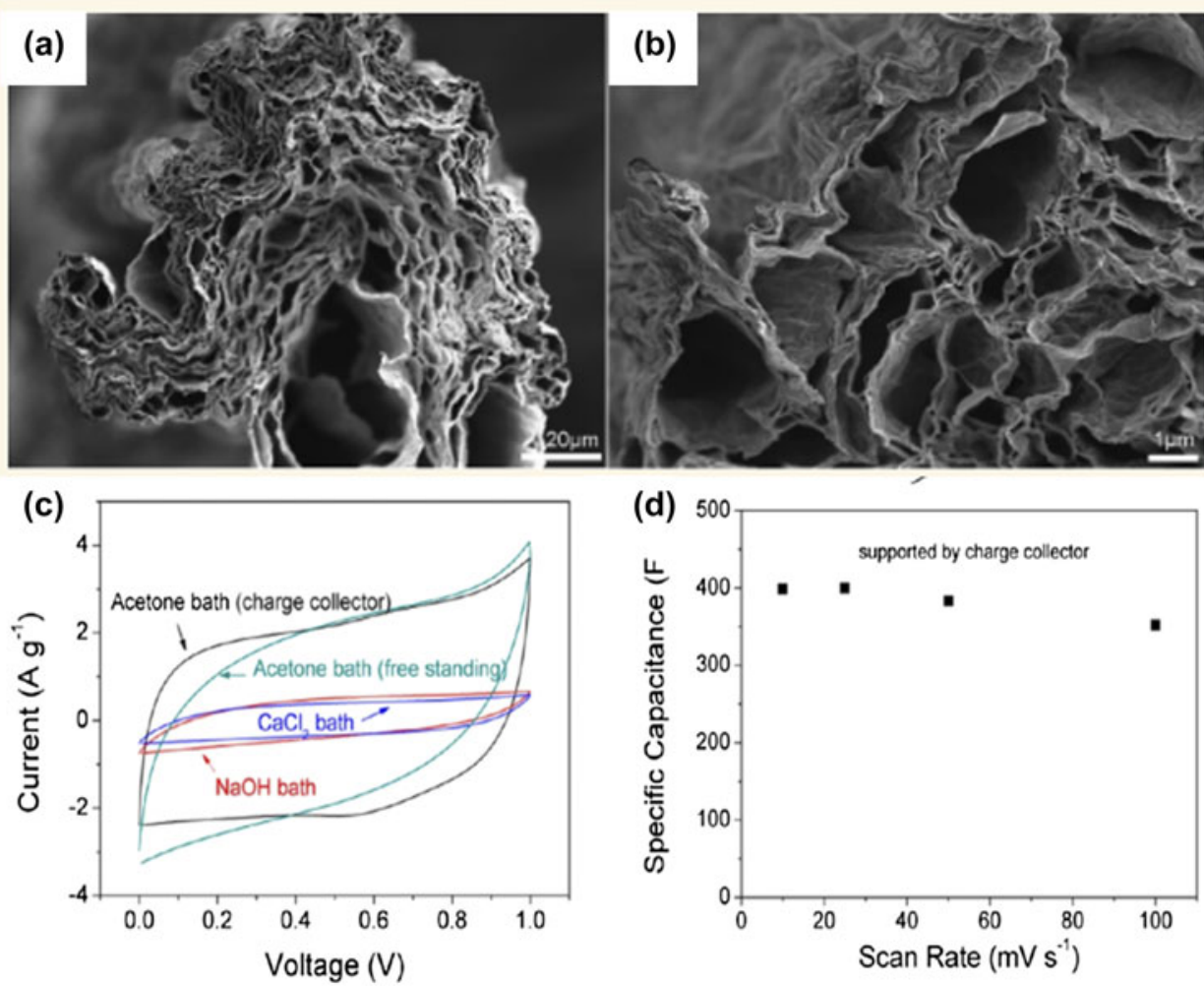

FIG. 5. (a and b) Cross section view of RGO fiber. (c) Cyclic voltammograms of heat-treated graphene fiber yarns produced in an acetone bath (both free-standing and deposited on charge collectors), alkaline bath $(\mathrm{NaOH})$, and ionic cross-linking using a divalent cation bath $\left(\mathrm{CaCl}_{2}\right)$ in $1 \mathrm{M}^{-}$ $\mathrm{H}_{2} \mathrm{SO}_{4}$ at $10 \mathrm{mV} / \mathrm{s}$. (d) Calculated specific capacitance of RGO fiber yarns fabricated in an acetone bath at various scan rates. Reprinted with permission from Ref. 73, Copyright 2014 American Chemistry Society. 
by direct deposition of $\mathrm{MnO}_{2}$ onto the surface of graphene fiber in the same group. ${ }^{76}$

\section{GRAPHENE AND CNT HYBRID ELECTRODES}

Recently, combining 1-D CNTs and 2-D graphene sheets to fabricate a composite film or fiber has attracted great attention since this method is efficient in utilizing the both merits of CNT and graphene and alleviating the aggregation and restacking of individual graphene sheets during the preparation process. ${ }^{77}$ CNTs serve as both conductive additives and structural pillows, which not only facilitate the infiltration of the electrolyte, but also improve the conductivity and electrochemical performance of the whole electrodes simultaneously. Simply by sonicating a mixture of GO dispersion and CNTs powder $(0.05 \mathrm{wt} \%$, respectively), a very stable and black suspension can be formed without visible sediment and the addition of any surfactants, indicating that GO acts as an amphiphilic molecule to realize the homogenous dispersion of CNTs. ${ }^{78}$

Compared with the conventional CNT-based and graphene-based fiber, graphene/CNT composite fibers for energy-storage devices show their unique mechanical and electrochemical properties. In the experiment reported by Sun et al., GO solution was added in the CNT array to form composite films, which were scrolled into fibers at the rotary rate of $2000 \mathrm{rpm} .{ }^{79} \mathrm{CNTs}$ function as effective bridges to improve the charge transport and decrease the contact resistances. The tensile strength of the graphene/
CNT composite fiber is $630 \mathrm{MPa}$. When used as supercapacitors, the fiber delivers a specific capacitances of up to $31.50 \mathrm{~F} / \mathrm{g}$. Similar coaxial wet-spun yarn supercapacitor (YSC) based on graphene and CNT was also reported by Gao' group. ${ }^{80}$ They demonstrated for the first time a coaxial wet-spinning assembly strategy to prepare core-sheath fibers. GO and CNT dispersions were first mixed with weight ratio of $1 / 1$ followed by transferring to an injection syringe connected with an inner core of spinneret. Sodium carboxymethyl cellulose was transferred to the outer channel of spinneret to form a novel coresheath structure (Fig. 6). The resulting compact two-ply YSCs show high capacitance and energy density values up to $269 \mathrm{mF} / \mathrm{cm}^{2}\left(239 \mathrm{~F} / \mathrm{cm}^{3}\right)$ and $5.91 \mathrm{~mW} \mathrm{~h} / \mathrm{cm}^{2}$ $\left(5.26 \mathrm{~mW} \mathrm{~h} / \mathrm{cm}^{3}\right)$ using liquid electrolyte.

In conclusion, an improved electrochemical performance of these hybrid electrodes was achieved due to the synergistic effect of both CNT and graphene. Graphene works as the main substrates with CNT as multifunctional pillows, which further enhance the conductivity, electrochemical capacity and mechanical properties of the whole system.

\section{OTHER CONDUCTIVE MATRIX BASED FLEXIBLE ELECTRODES}

Despite the progress made in flexible electrodes by using CNT and graphene as both active materials and current collectors, other forms of carbon materials and
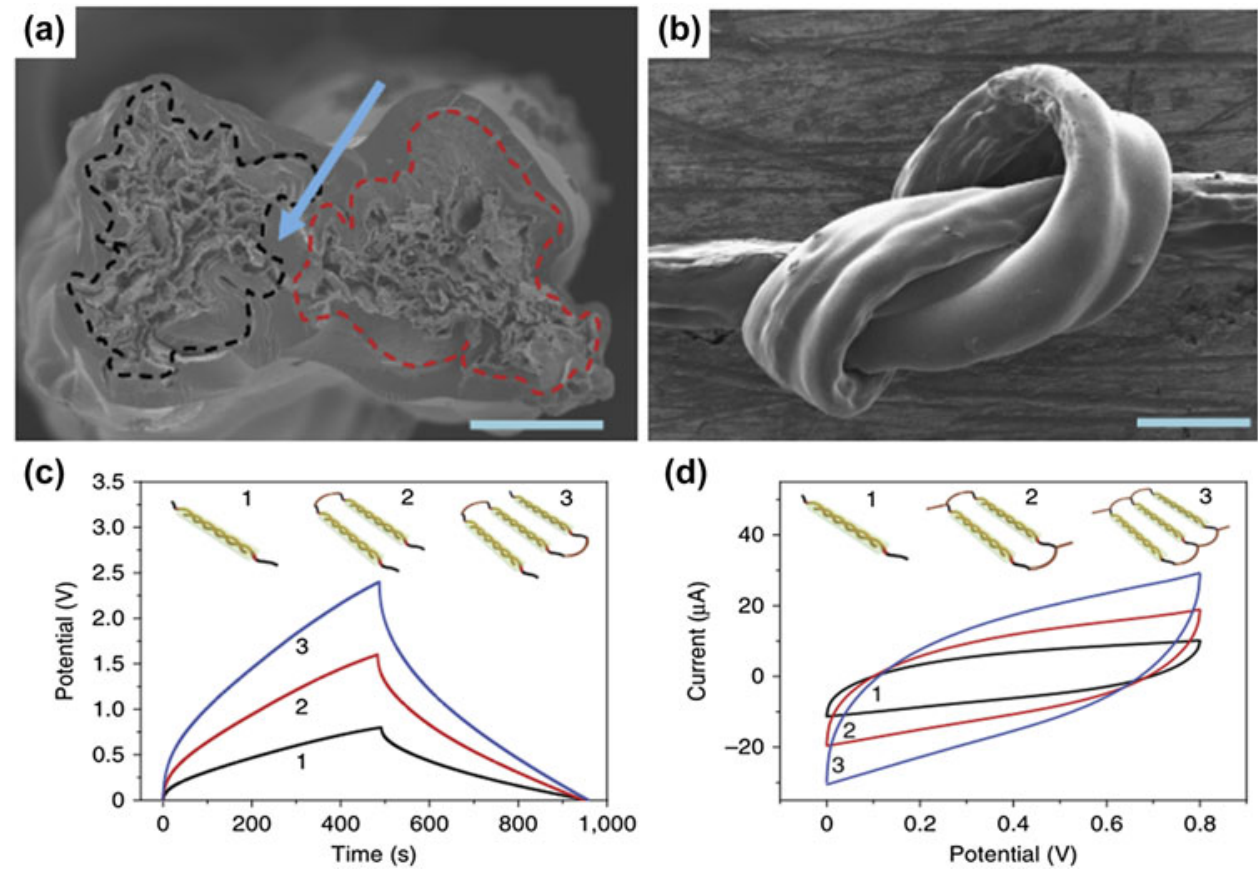

FIG. 6. (a) Scanning electron microscope (SEM) images of cross-sectional view of a two-ply YSC. (b) SEM image of a two-ply YSC knot. (c) Galvanostatic charge and discharge curves of single, two and three YSCs connected in series. (d) CV curves of single, two and three YSCs connected in parallel, and the insets show the corresponding schematics (at a scan rate of $10 \mathrm{mV} / \mathrm{s}$ ). Reprinted with permission from Ref. 80 , Copyright 2014, Nature Publishing Group. 
some novel mental-based current collectors are also widely explored to achieve flexible electrodes with excellent properties.

It has been demonstrated in previous discussion that the surface condition of the active materials greatly influence their electrochemical properties. Carbon cloth-based solid-state supercapacitor was prepared by Wang et al. via the modification of carbon fiber. ${ }^{81}$ They reported an effective chemical oxidation and reduction method to increase surface area of the carbon materials. $\mathrm{HNO}_{3}$, $\mathrm{H}_{2} \mathrm{SO}_{4}$ and $\mathrm{KMnO}_{4}$ were used as oxidizing reagents to modify the surface of carbon cloth and form a rough surface. Figure 7 shows a core-shell structure at the edge of each carbon fiber. Then the modified carbon fibers were reduced by hydrazine and ammonia. PVA and $\mathrm{H}_{2} \mathrm{SO}_{4}$ were used to prepare the solid-state electrolyte. The symmetric solid state supercapacitor device exhibits a capacitance of $15.3 \mathrm{mF} / \mathrm{cm}^{2}$ with a rate retention of $76 \%$ at $10 \mathrm{mV} / \mathrm{s}$ achieved at $1000 \mathrm{mV} / \mathrm{s}$.

Carbon cloth can also be used as conductive substrates with active materials deposited on the surface of it. $\mathrm{MoS}_{2}$ nanoflakes were grown on the surface of carbon fiber and used as flexible LIB anode. ${ }^{82}$ One-step hydrothermal synthesis method was adopted to prepare hierarchical $\mathrm{MoS}_{2}$ flakes with $\mathrm{MoO}_{3}$, thioacetamide, and urea as precursors. When used as a flexible LIB anode, the flexible 3-D $\mathrm{MoS}_{2}$ has a high reversible capacity of $3.0-3.5 \mathrm{~mA} \mathrm{~h} / \mathrm{cm}^{2}$ at a current density of $0.15 \mathrm{~mA} / \mathrm{cm}^{2}$ and outstanding rate stability. Similarly, Liu et al. reported a flexible anode with $\mathrm{ZnCo}_{2} \mathrm{O}_{4}$ nanowire uniformly deposited on carbon fiber (see Fig. 8). ${ }^{83}$ A two-step process was needed to synthesize hierarchical $\mathrm{ZnCo}_{2} \mathrm{O}_{4}$ nanowire. The binder-free anodes deliver a high reversible capacity of $1300-1400 \mathrm{~mA} \mathrm{~h} / \mathrm{g}$ and excellent cycling ability even after 160 cycles with a capacity of $1200 \mathrm{~mA} \mathrm{~h} / \mathrm{g}$.

In 2005, a new type of carbon nanofiber with novel bamboo-like structure was reported and shows excellent mechanical flexibility, foldability and electrochemical performances. ${ }^{84}$ Polyacrylonitrile and tetraethyl orthosilicate in dimethylformamide were used to make the nanofibers by electrospinning. After heating the precursors at $1200{ }^{\circ} \mathrm{C}$ in a $\mathrm{H}_{2} / \mathrm{Ar}$ atmosphere, ultrafine $\mathrm{SiO}_{2}$ clusters aggregate into much larger $\mathrm{SiO}_{2}$ particles, which were etched by $\mathrm{HF}$ aqueous solution to generate interior holes. In the fiber, an even distribution of the holes not only enhances its mechanical stability, but also reduces ion diffusion resistance. The specific power and energy densities of the nanofibers are $61.3 \mathrm{~kW} / \mathrm{kg}$ and $2.37 \mathrm{Wh} / \mathrm{kg}$, respectively.

As 1-D conductive substrates, metal wires such as $\mathrm{Ni}$ and $\mathrm{Ti}$ fibers, are typically explored for efficient electrode materials in a variety of energy-storage devices due to their high electrical conductivity. Wu et al. demonstrated a $\mathrm{ZnCo}_{2} \mathrm{O}_{4}$ nanorod based flexible supercapacitor with $\mathrm{Ni}$ wire as current collector and flexible substrate. ${ }^{85} \mathrm{~A}$ simple and rapid one-step hydrothermal process was taken in the preparation of the electrode. The result shows that, 97.9\% and $92 \%$ of the initial capacitance are maintained after 800 times cycles at varied current and 3500 charge/discharge cycles, respectively.

Nickel foam can not only be used as templates to synthesize GF with interconnected network, but also can serve as highly conductive and 3-D scaffold for flexible batteries. The unique structure of nickel foam play an important role in reducing the diffusion resistance of electrolytes and enhancing ion transportation by providing very smooth electron pathways. ${ }^{86} \mathrm{Up}$ to now, nickel foam has been mostly reported in many literature and used as flexible current collector. Liu et al. reported that nickel foam can be used to offer flexible room for the accommodation of volume expansion of $\mathrm{Si}^{87} \mathrm{Si}$ nanowires (SiNWs) were dispersed in water with SDBS as surfactant. Then Ni foam was immersed in the SiNWs solution for $30 \mathrm{~min}$ followed by pressing at $4 \mathrm{MPa}$ CR2032 coin was assembled to test the electrode and it
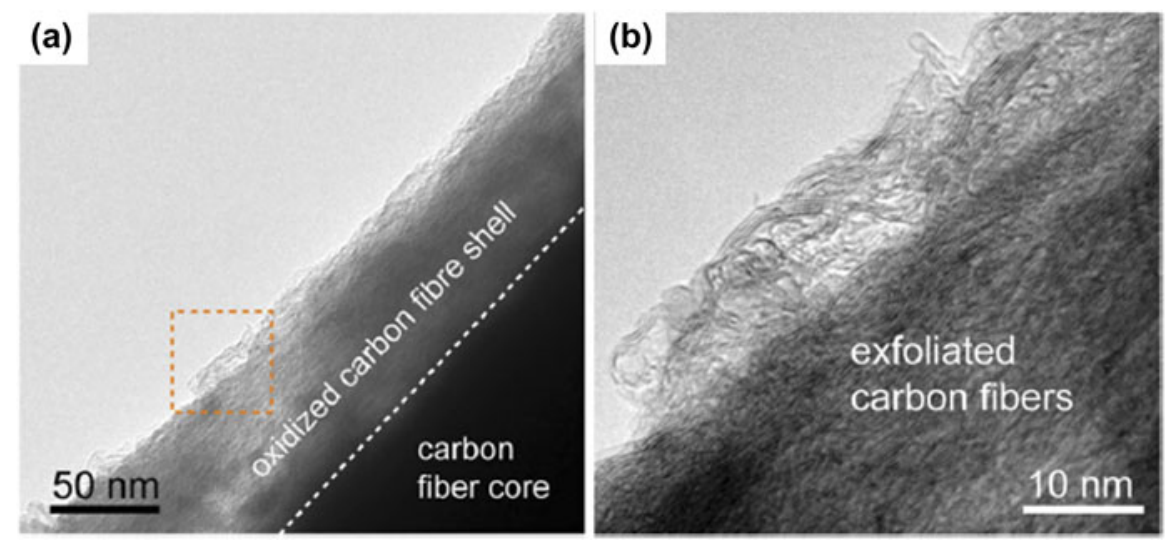

FIG. 7. (a) Low-magnification TEM image collected at the edge of the oxidized carbon fiber. (b) High-resolution TEM image showing the exfoliated carbon fibers. Reprinted with permission from Ref. 81, Copyright 2014 WILEY-VCH Verlag GmbH \& Co. KGaA, Weinheim. 


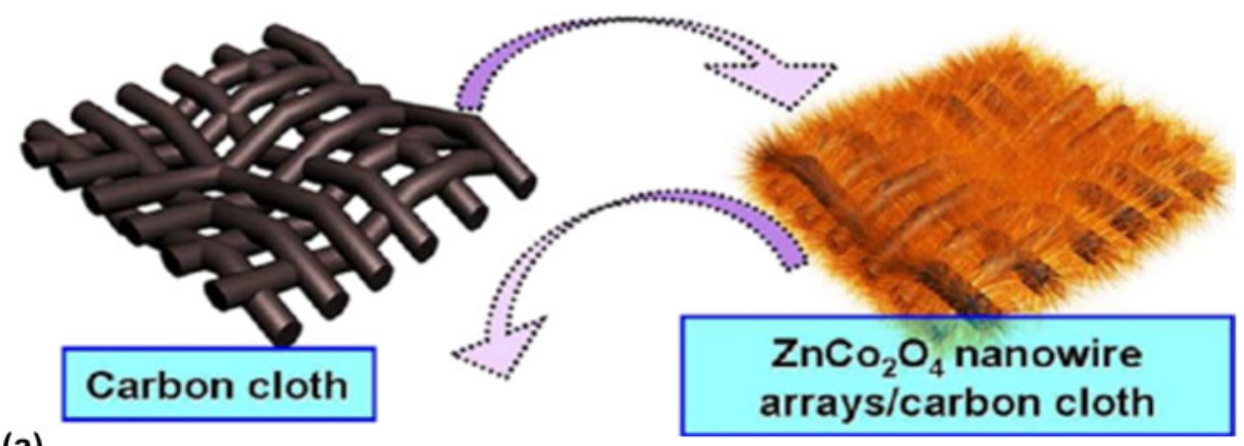

(a) $\mathrm{ZnCO}_{2} \mathrm{O}_{4}$ nanowire
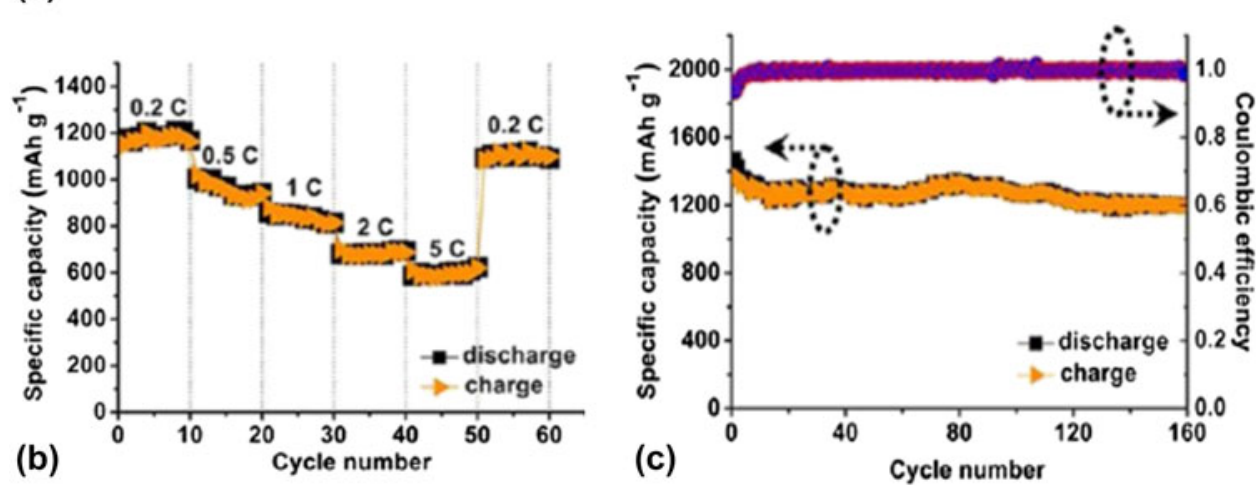

FIG. 8. (a) Schematic illustration of the synthesis of flexible 3-D $\mathrm{ZnCo}_{2} \mathrm{O}_{4}$ nanowire arrays/carbon cloth. (b) Capacity versus cycle number plot at different charging rates. (c) Long-term cycling of the $\mathrm{ZnCo}_{2} \mathrm{O}_{4}$ nanowire arrays/carbon cloth electrode with a reversible capacity value of $1200 \mathrm{~mA} \mathrm{~h} / \mathrm{g}^{1}$ after 160 cycles with Coulombic efficiency of 99\%. Reprinted with permission from Ref. 82, Copyright 2014 Royal Society of Chemistry.

achieves an initial capacity of as high as $2486 \mathrm{~mA} \mathrm{~h} / \mathrm{g}$ with a reversible capacity of $1500 \mathrm{~mA} \mathrm{~h} / \mathrm{g}$ retained even after 50 cycles of charging and discharging.

Due to the high conductivity and 3-D interconnected structure of nickel foam, it is one of the most optimal substrates for electrodeposition methods with various nanomaterials deposited on the surface of it and used as active materials. Yang et al. reported a supercapacitors by electrodepositing $\mathrm{Ni}(\mathrm{OH})_{2}$ on nickel foam with. ${ }^{86}$ The electrode was tested in the potential range of $0.05-0.45 \mathrm{~V}$ with a maximum specific capacitance as high as $3152 \mathrm{~F} / \mathrm{g}$ in $3 \% \mathrm{KOH}$ solution at a charge/discharge current density of $4 \mathrm{~A} / \mathrm{g}$. The enhancement of the specific capacitance is mainly attributed to the even distribution of $\mathrm{Ni}(\mathrm{OH})_{2}$ on the surface of conductive $\mathrm{Ni}$ substrate and the intimate contact between the active materials and the conductive substrate. Similar electrodepositing method was also applied by Chen's group, as shown in Fig. 9. ${ }^{88}$ They reported a high-performance cathode for $\mathrm{Li}-\mathrm{S}$ batteries with nickel foam as a flexible and binder-free current collector. Electrodepostion method was applied to prepare reactive sulfur nanodots with average size of $2 \mathrm{~nm}$, which are uniformly distributed on the surface of $\mathrm{Ni}$ foam. Sulfur content could be controlled from 0.21 to $4.79 \mathrm{mg} / \mathrm{cm}^{2}$ simply by controlling the deposition time. The electrode delivers a high initial discharge capacity (1458 $\mathrm{mA} \mathrm{h/g}$ at $0.1 \mathrm{C})$, high rate capability
$(521 \mathrm{~mA} \mathrm{~h} / \mathrm{g}$ at $10 \mathrm{C})$ and long cycling stability $(528 \mathrm{~mA} \mathrm{~h} / \mathrm{g}$ after 1400 cycles at $5 \mathrm{C})$.

\section{NONCONDUCTIVE SUBSTRATES}

Compared with the conductive substrates, other nonconductive substrates with inherent advantages in flexibility, mechanical robustness and porosity, such as commonly used separators, ${ }^{89}$ PDMS, ${ }^{90}$ poly $(m$-phenylene isophthalamide), ${ }^{91}$ textiles $^{92}$ and other polymers, have also been widely applied in the flexible electrodes to obtain high performance flexible and bendable electrodes.

Commercially used separator with inherent flexibility is an indispensable part of the whole LIB system to prevent short circuit, so the energy density will not be sacrificed when they are used as flexible substrates. Celgard 3500 have been explored by Li et al. to fabricate a binder-free and current collector-free anode. ${ }^{89}$ Entangled CNTs were trapped on the surface of the separator after filtration and served as active materials. Based on this concept, Zhou et al. designed a novel sandwich structure with pure sulfur trapped in two membranes. ${ }^{93}$ One graphene membrane was used as a current collector (GCC) with pure sulfur coated on it as active materials. To further improve the mechanical robustness and flexibility of the electrode, a commercial polymer separator (G-separator) was coated with another graphene layer and 


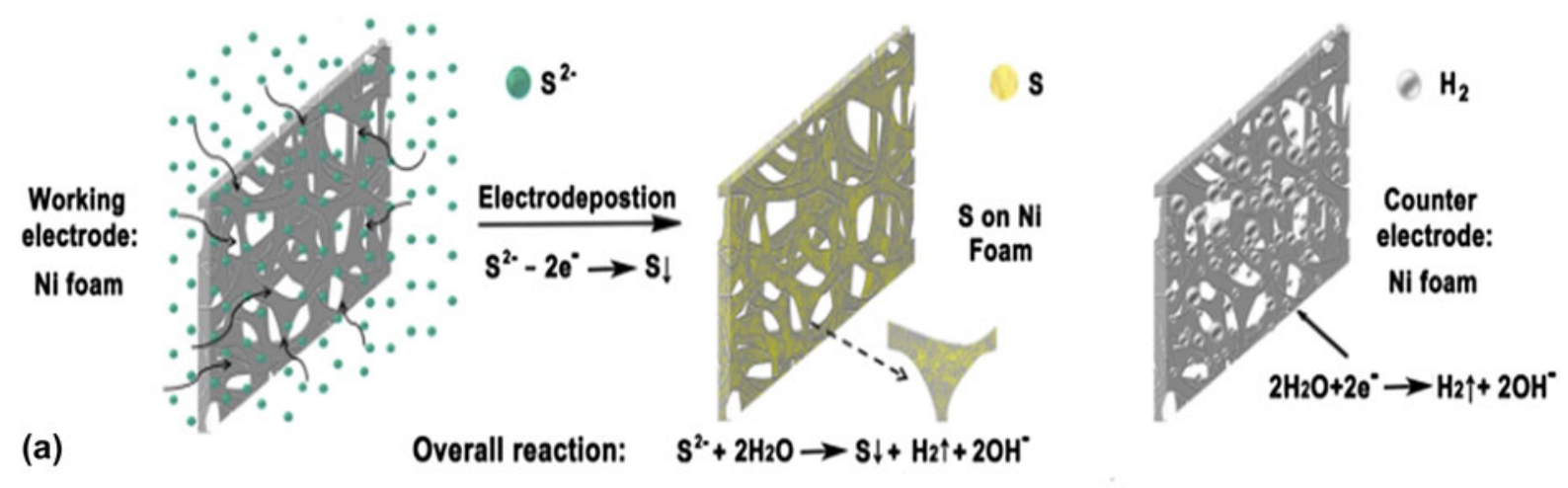

(b)
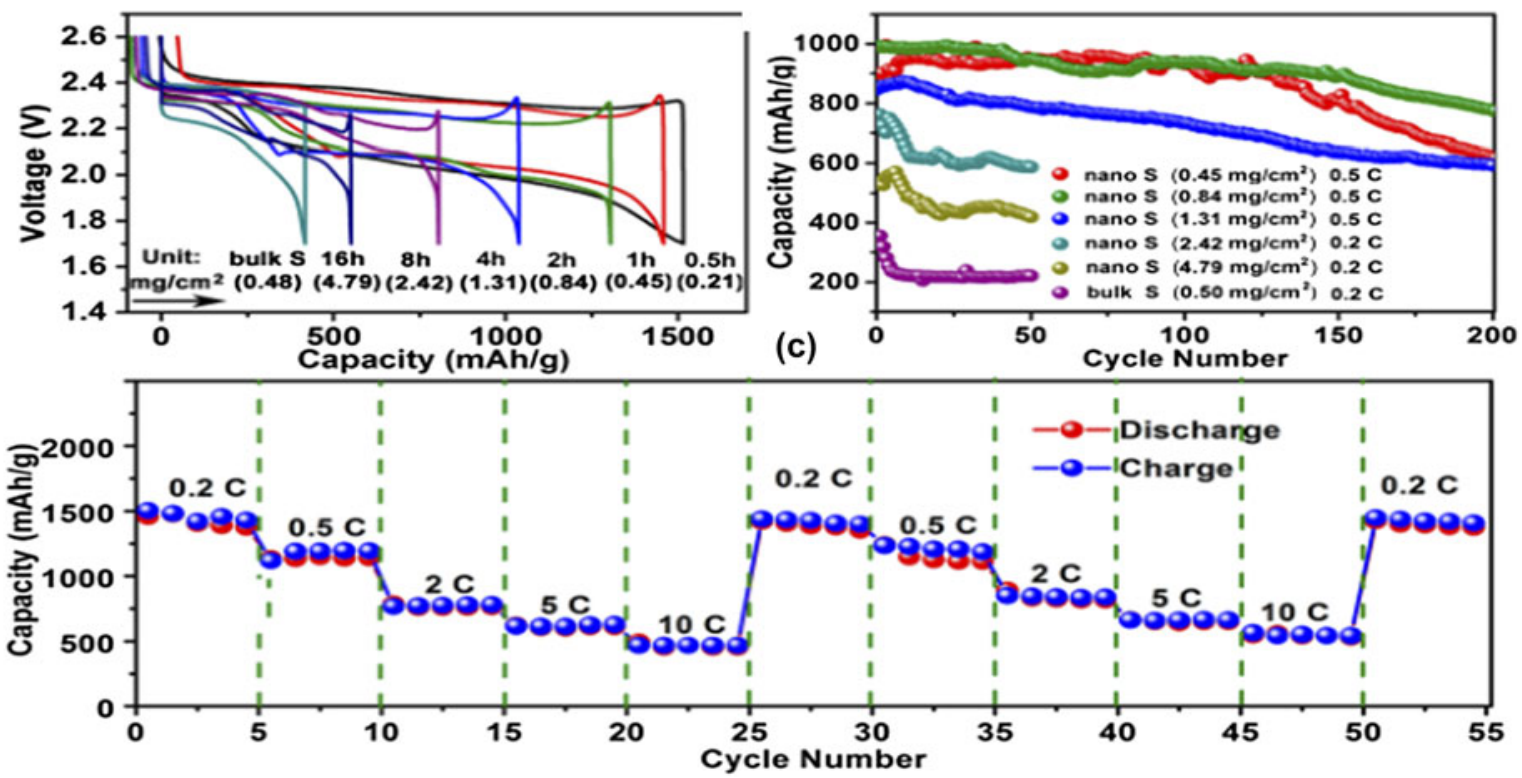

FIG. 9. (a) A schematic diagram of preparing sulfur nanodots on Ni foam with an electrodeposition method. (b) Discharge and charge profiles at 0.1 C. (c) Cycling performance of $\mathrm{S}$ nanodots cathodes with different $\mathrm{S}$ content and bulk $\mathrm{S}$ cathode (without the addition of $\mathrm{Li}_{2} \mathrm{~S}_{8}$ ). (d) $\mathrm{Rate}$ performance of S nanodots cathodes. Reprinted with permission from Ref. 88, Copyright 2015 American Chemical Society.

used as both separator and current collector as shown in Fig. 10. The excellent flexibility of the electrodes should be attributed to the mechanical robustness of both polymer separator and graphene film.

From the above discussion, we know that graphene films and foams are widely used as flexible current collectors for LIBs and ECs. However, the mechanical properties of the graphene-based materials limit their further application. In addition to the separators mentioned before, PDMS is used to further improve the flexibility of graphene films and foams via different methods. Zhou et al. developed a flexible Li-S battery electrode by using GF as a current collector and host for large sulfur loading via a simple slurry infiltration. ${ }^{94}$ The PDMS coated on the surface of GF makes the interconnected network sufficiently robust, ensuring the flexibility of the cathode. The high sulfur-loaded electrodes retain a high rate performance of $450 \mathrm{~mA} \mathrm{~h} / \mathrm{g}$ under a large current density of $6 \mathrm{~A} / \mathrm{g}$ and preserve stable cycling performance with $0.07 \%$ capacity decay per cycle over 1000 cycles, as shown in Fig. 11.

In addition to the hosts and substrates for graphenebased materials, PDMS is also able to be used as transparent and stretchable substrate for CNT. A highly aligned carbon nanotube sheets with excellent optical transmittance and mechanical stretchability was developed by Chen et al. as all-solid supercapacitors. ${ }^{95}$ Vertically aligned CNT was synthesized by CVD with ethylene as the carbon source followed by directly drawing the horizontally aligned CNT sheet onto a PDMS substrate. The transparent supercapacitor exhibits a specific capacitance of $7.3 \mathrm{~F} / \mathrm{g}$ and can be stretched up to $30 \%$ strain without any obvious structural deformation and capacity decay even over hundreds of stretching cycles. To further utilize the unique properties of PDMS, a porous, stretchable, and conductive PDMS-based electrodes embedded with carbon CNTs was reported by Lee et al. for LIBs. ${ }^{96}$ In their experiment, polymethylmethacrylate was added 

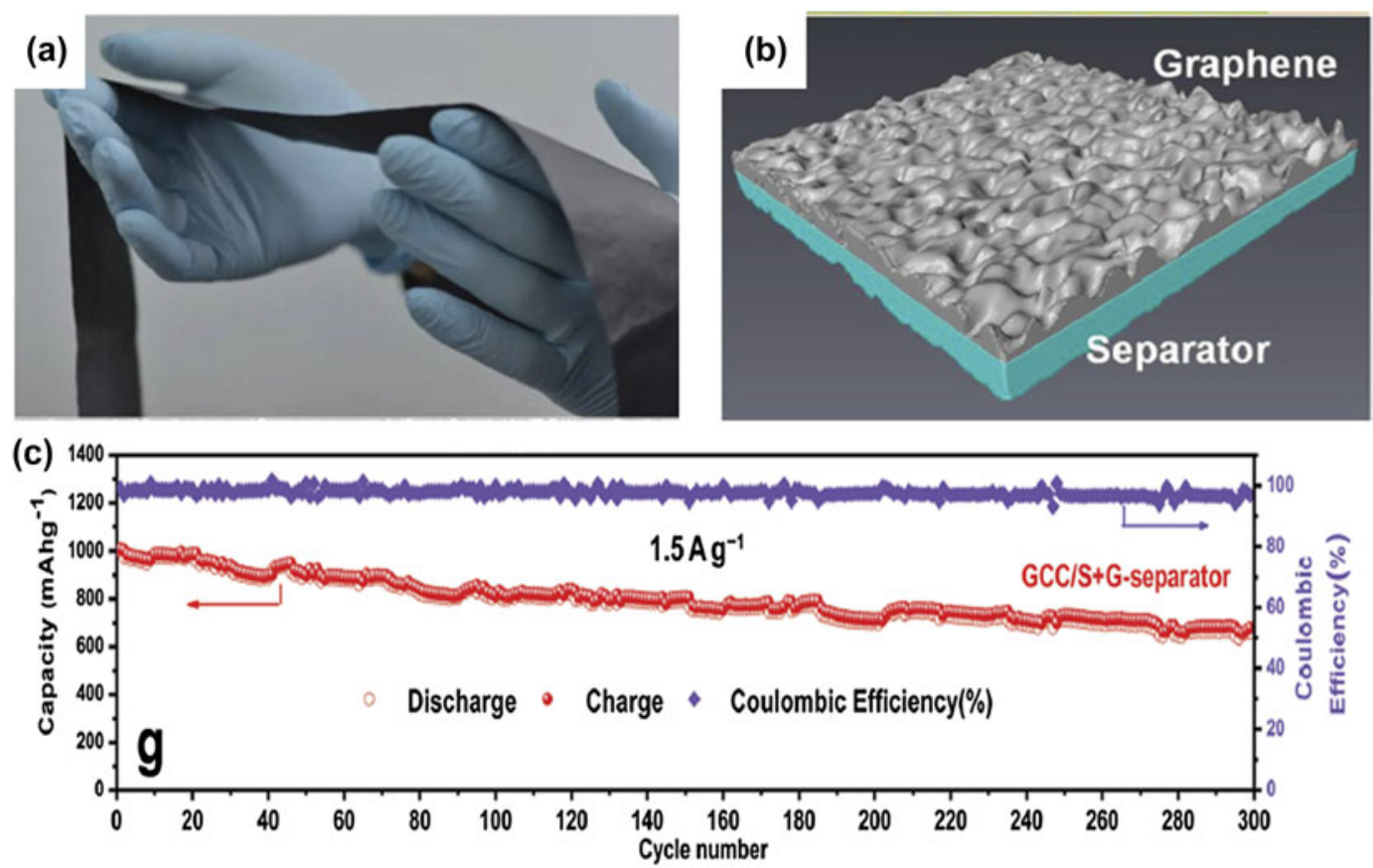

FIG. 10. (a) Photographs of an as-prepared large-area GCC strip (width: $6 \mathrm{~cm}$ ) with good flexibility. (b) 3-D reconstructed G-separator. (c) Cycling stability of the Li-S batteries with GCC/S+G-separator at $1.5 \mathrm{~A} / \mathrm{g}^{1}$ for 300 cycles. Reprinted with permission from Ref. 93, Copyright 2014 WILEY-VCH Verlag GmbH \& Co. KGaA, Weinheim.

and then removed to generate well-controlled pore networks. It has been demonstrated that the porous nature of the nanocomposites plays an important role in controlling the infiltration and accessibility of the electrolyte. As a result, an improvement of $670 \%$ in capacity of the porous nanocomposite has been achieved compared to that of a nonporous nanocomposite.

Despite the advanced performance for flexible LIBs and ECs, most electrode materials are only synthesizable in certain nanostructures or carbon-based templates, which may bring about high cost and manufacturing complexity. Textile and cellulose are naturally abundant resource and have potential applications in future wearable electronics due to their mechanical strength and low cost. Hu et al. reported wearable power devices based on natural textiles using an extremely simple "dipping and drying" process. ${ }^{97}$ SDBS was used as surfactant to form well-dispersed SWNTs. Then a textile was dipped into the suspension and subsequently dried in an oven. The textile with a conductivity of $125 \mathrm{~S} / \mathrm{cm}$ and sheet resistance less than $1 \Omega /$ sq was produced. They also demonstrated that the loading of pseudocapacitor materials $\mathrm{MnO}_{2}$ could be achieved by electrodeposition.

Similarly, natural mica was used as sacrificial substrates for flexible all-solid LIBs. Koo et al. reported a new universal transfer approach with $\mathrm{LiCoO}_{2}$, lithium phosphorus oxynitride and a lithium metal as cathode, solid electrolyte and anode, respectively. ${ }^{98}$ Packaged in PDMS, the thin-film LIB is capable of a maximum $4.2 \mathrm{~V}$ charging voltage and $106 \mu \mathrm{A} \mathrm{h} / \mathrm{cm}^{2}$ capacity. Meanwhile, the battery could operate a flexible organic LED system when integrated into a single platform, which indicate the high performance for flexible LIBs, as shown in Fig. 12.

From the above discussion, it can be concluded that, due to the excellent mechanical properties and chemical stability of PDMS, it is widely used in various energystorage devices and serves as stretchable and transparent substrates for LIBs and ECs. Meanwhile, some natural materials have gradually attracted increasing attention as emerging substrates for flexible LIBs and ECs.

\section{ELECTROLYTES}

As a key component of LIBs or ECs, the selection of suitable electrolytes has a great influence on both the performance and design of the flexible LIBs and ECs. Conventionally, the electrolyte serves as both Li-ion diffusion media and insulator to prevent direct contact between the two electrodes in the system. In flexible electrodes, the electrolytes should possess more properties such as high ionic conductivity, good mechanical robustness, low risk of electrolyte leakage and low toxicity.

Electrolytes are commonly divided into liquid electrolytes and solid electrolytes. In terms of ionic conductivity and the physical contact between the electrodes and the electrolytes, liquid electrolytes exhibit their advantages over that of solid electrolytes. So they are mostly used in the existing LIBs or ECs. However, after repeated deformation or bending, there is a higher risk of the leakage of liquid electrolytes and the related safety concerns such 


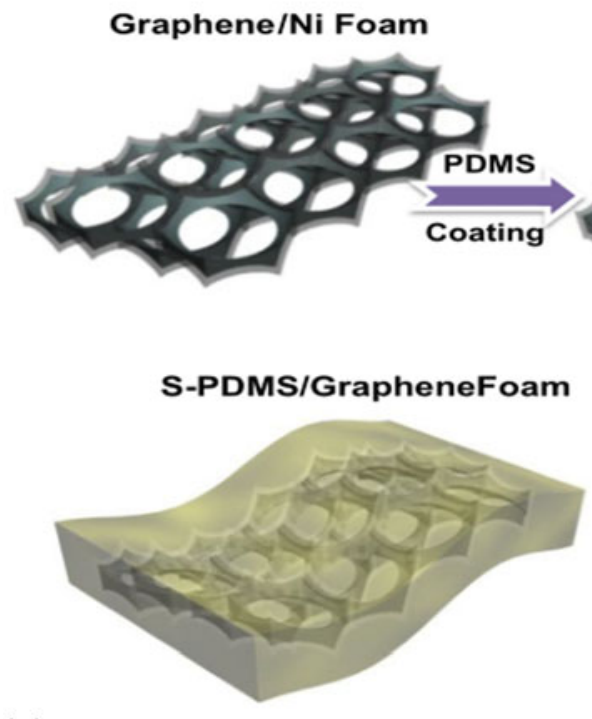

PDMS/Graphene/Ni Foam

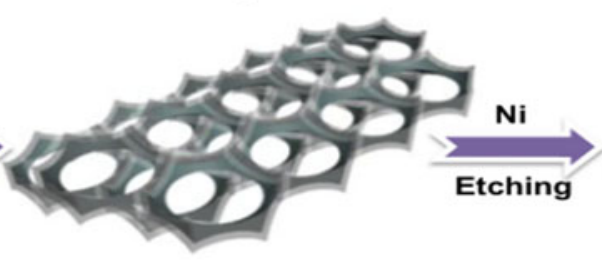

S-PDMS/GrapheneFoam

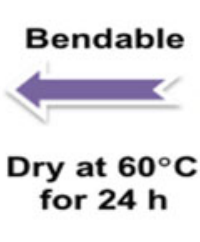

PDMS/GrapheneFoam

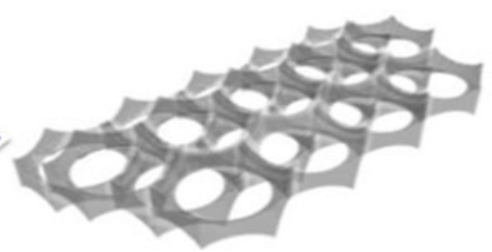

Fill in Well-Mixed Sulfur Slurry

(a)
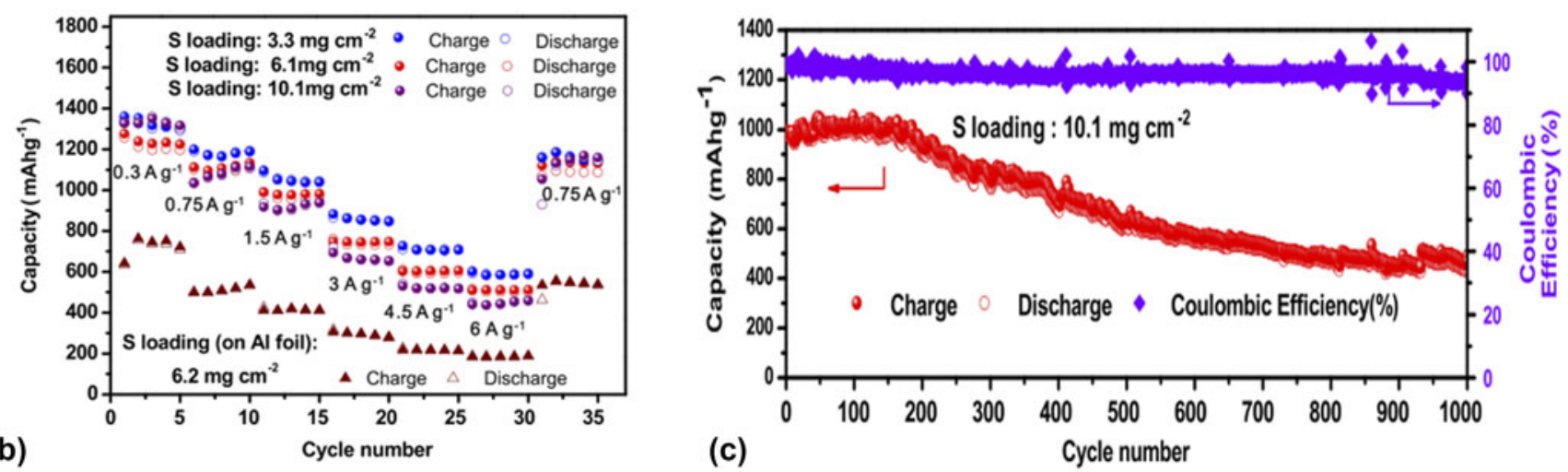

FIG. 11. (a) Schematic of the procedure for fabricating PDMS/GF and S-PDMS/GF electrodes. (b) Rate performance of the S-PDMS/GF electrodes with different sulfur loadings. (c) Cycling performance an Coulombic efficiency of the S-PDMS/GF electrode with a $10.1 \mathrm{mg} / \mathrm{cm}^{2}$ sulfur loading at $1500 \mathrm{~mA} \mathrm{~h} / \mathrm{g}$ for 1000 cycles. Reprinted with permission from Ref. 94, Copyright 2015 Elsevier Ltd.

as flammability, toxicity and explosion. As a result, elaborate packaging and assembly of these cells are highly required to prevent the possible short-circuit and potential dangers.

Solid-state electrolytes, especially the shape-comfortable solid-state electrolytes discussed in this essay, are considered to be safer and more reliable than liquid organic electrolytes because of their chemical stability, low metallic corrosion and minimal risk of electrolyte leakage. However, the practical applications of the conventional solid-state electrolytes were hindered by its low ionic conductivity, brittleness and narrow electrochemical window stability. ${ }^{99}$ The breakthrough was made in plastic crystal electrolytes (PCEs) and gel-polymer electrolytes (GPEs). For example, Ha et al. ${ }^{100}$ demonstrated a facile approach based on integration of a UV (ultraviolet)-curable semiinterpenetrating polymer network (semi-IPN) matrix with a PCE to achieve improved mechanical bendability and electrochemical performance. In addition, no decomposition of any components in the plastic crystal composite electrolyte (PCCE) takes place below $5.0 \mathrm{~V}$ versus $\mathrm{Li}^{+} / \mathrm{Li}$, indicating stable electrochemical window of the PCCEs. Incorporating liquid electrolytes into polymer matrix also forms solid-state electrolytes with improved mechanical properties. Recently, a facile and scalable approach was reported by Kil et al. in the same group to prepare highly ion-conductive and bendable polymer electrolytes. ${ }^{101}$ The GPE is composed of a UV-cured polymer matrix, high-boiling point liquid electrolyte with alumina $\left(\mathrm{Al}_{2} \mathrm{O}_{3}\right)$ nanoparticles used as a functional filler to control the rheological properties of the electrolyte mixture.

\section{CONCLUSION AND FUTURE PROSPECTS}

This review summarized and discussed the recent progresses made in flexible electrodes for LIBs and ECs, with a focus on different flexible active materials and substrates. As lightweight, thin and flexible matrix, 
(a)

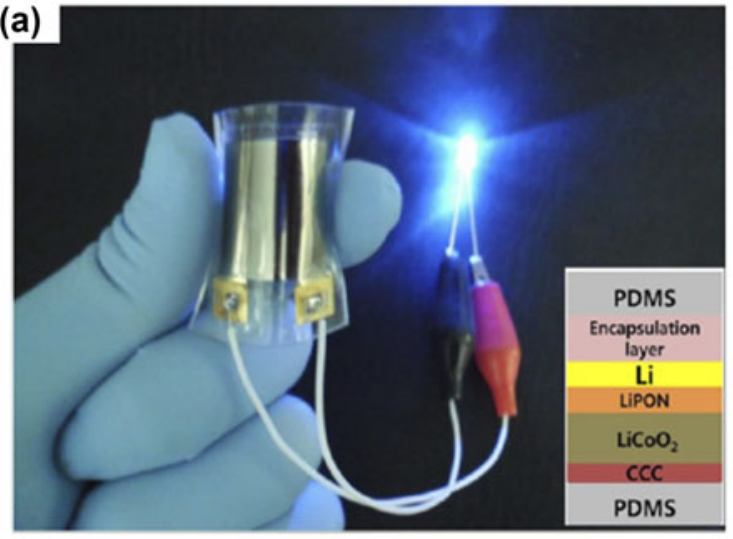

(c)

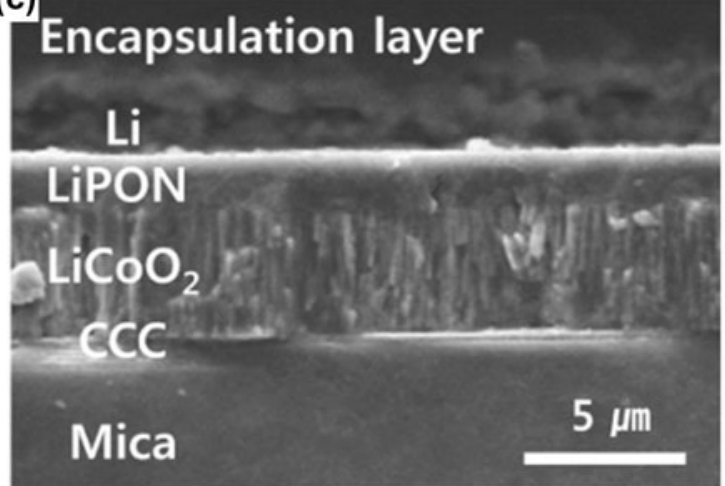

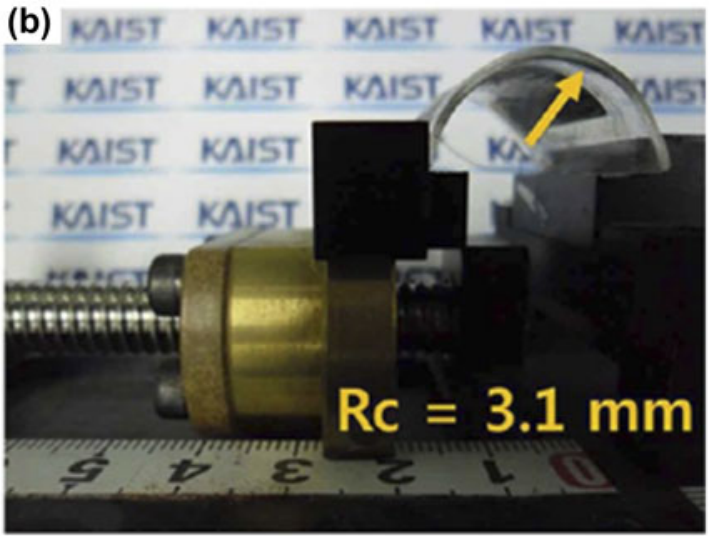

(d)

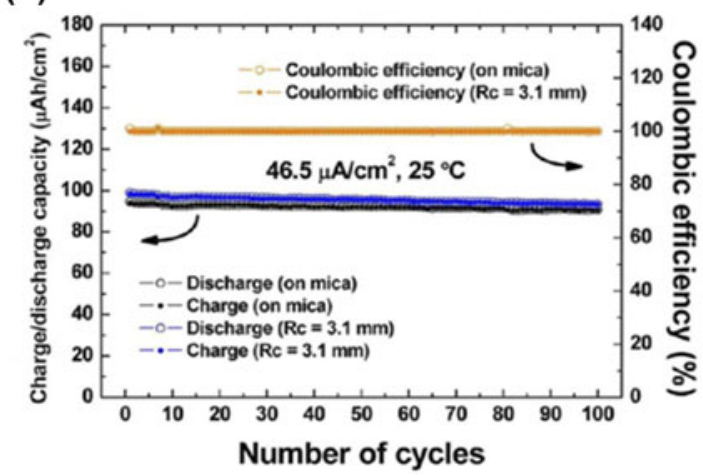

FIG. 12. (a) Photograph of a bendable LIB turning on a blue LED in bent condition. (b) Robustness tests of a flexible LIB on a bending stage machine. (c) Cross-sectional SEM image of a thin-film LIB. (d) Coulombic efficiencies of a flexible LIB bent to $R_{\mathrm{c}}=3.1 \mathrm{~mm}$ and a mica LIB Reprinted with permission from Ref. 98, Copyright 2012 American Chemical Society.

carbon-based materials such as CNT, graphene and carbon fibers are still the dominant materials that are used as active materials for flexible electrodes in LIBs and ECs. However, the weaknesses of these materials lie in its low reversible capacity and low Coulombic efficiency, which hinders its practical applications and commercialization. When used as flexible substrates and embedded-in current collectors, the carbon-based materials show excellent mechanical strength and chemical stability. In addition, novel metal foams/fibers, natural materials and some nonconductive polymers are also widely adopted as mechanically robust substrates and exhibit great potential in the further utilization.

To achieve scalable and low-cost production of flexible energy-storage devices such as LIBs and ECs in the near future, improvements can be made in the reduction of the costs of flexible substrates, the selection of suitable electrolytes and simplification of the fabrication process. Some safety concerns should also be addressed by optimizing the preparation parameters, electrolytes along with the packaging materials. Meanwhile, improving the mechanical strength of both the electrodes and electrolyte is necessary to further realize the applications of these flexible LIBs and ECs in roll-up displays and wearable devices.
At the same time, as novel electrochemical nanomaterials in the area of energy-storage devices, comprehensive measurement standards and assessment criterions are needed for both the electrochemical and mechanical performance of these flexible electrodes. For example, the criterions should involve some necessary factors such as the intensity and types of deformation, bending time and radii, stretchability and mechanical wearability to accurately and systematically characterize the flexible properties of the electrodes.

Finally, various methods need to be explored to search for novel materials with both excellent mechanical and electrochemical properties. Meanwhile, increasing the mass loading of these active materials on the flexible electrodes is urgently required to further improve its energy density of the electrodes without compromising the volumetric energy density.

As the era of smart electronics and next-generation flexible energy storage and conversion systems has come, flexible electronics market will continue to grow. Despite the challenges discussed above in this essay, the unique advantages and properties of the flexible electrodes will be further explored to meet increasing demands in flexible electronics market. 


\section{ACKNOWLEDGMENT}

This work Supported by Natural Science Foundation of Shanxi Province (Grant No: 2015021062).

\section{REFERENCES}

1. M. Armand and J-M. Tarascon: Building better batteries. Nature 451(7179), 652 (2008).

2. M. Yazici, D. Krassowski, and J. Prakash: Flexible graphite as battery anode and current collector. J. Power Sources 141(1), 171 (2005)

3. S. Chen, Y. Xin, Y. Zhou, Y. Ma, H. Zhou, and L. Qi: Self-supported $\mathrm{Li}_{4} \mathrm{Ti}_{5} \mathrm{O}_{12}$ nanosheet arrays for lithium ion batteries with excellent rate capability and ultralong cycle life. Energy Environ. Sci. 7(6), 1924 (2014).

4. A.S. Arico, P. Bruce, B. Scrosati, J-M. Tarascon, and W. Van Schalkwijk: Nanostructured materials for advanced energy conversion and storage devices. Nat. Mater. 4(5), 366 (2005).

5. Y. Hu and X. Sun: Flexible rechargeable lithium ion batteries: Advances and challenges in materials and process technologies. J. Mater. Chem. A 2(28), 10712 (2014).

6. K. Wang, S. Luo, Y. Wu, X. He, F. Zhao, J. Wang, K. Jiang, and S. Fan: Super-aligned carbon nanotube films as current collectors for lightweight and flexible lithium ion batteries. Adv. Funct. Mater. 23(7), 846 (2013).

7. B.J. Landi, M.J. Ganter, C.D. Cress, R.A. DiLeo, and R.P. Raffaelle: Carbon nanotubes for lithium ion batteries. Energy Environ. Sci. 2(6), 638 (2009).

8. Y.A. Kim, M. Kojima, H. Muramatsu, S. Umemoto, T. Watanabe, K. Yoshida, K. Sato, T. Ikeda, T. Hayashi, and M. Endo: In situ Raman Study on single-and double-walled carbon nanotubes as a function of lithium insertion. Small 2(5), 667 (2006).

9. C. Garau, A. Frontera, D. Quiñonero, A. Costa, P. Ballester, and P.M. Deyà: Ab initio investigations of lithium diffusion in single-walled carbon nanotubes. Chem. Phys. 297(1), 85 (2004).

10. H. Gwon, J. Hong, H. Kim, D-H. Seo, S. Jeon, and K. Kang: Recent progress on flexible lithium rechargeable batteries. Energy Environ. Sci. 7(2), 538 (2014).

11. S.Y. Chew, S.H. Ng, J. Wang, P. Novák, F. Krumeich, S.L. Chou, J. Chen, and H.K. Liu: Flexible free-standing carbon nanotube films for model lithium-ion batteries. Carbon 47(13), 2976 (2009).

12. B.J. Landi, M.J. Ganter, C.M. Schauerman, C.D. Cress, and R.P. Raffaelle: Lithium ion capacity of single wall carbon nanotube paper electrodes. J. Phys. Chem. C 112(19), 7509 (2008)

13. D. Tasis, N. Tagmatarchis, A. Bianco, and M. Prato: Chemistry of carbon nanotubes. Chem. Rev. 106(3), 1105 (2006).

14. I. Mukhopadhyay, N. Hoshino, S. Kawasaki, F. Okino, W. Hsu, and $\mathrm{H}$. Touhara: Electrochemical $\mathrm{Li}$ insertion in B-doped multiwall carbon nanotubes. J. Electrochem. Soc. 149(1), A39 (2002).

15. S.W. Lee, N. Yabuuchi, B.M. Gallant, S. Chen, B-S. Kim, P.T. Hammond, and Y. Shao-Horn: High-power lithium batteries from functionalized carbon-nanotube electrodes. Nat. Nanotechnol. 5(7), 531 (2010).

16. H.X. Zhang, C. Feng, Y.C. Zhai, K.L. Jiang, Q.Q. Li, and S.S. Fan: Cross-stacked carbon nanotube sheets uniformly loaded with $\mathrm{SnO}_{2}$ nanoparticles: A novel binder-free and highcapacity anode material for lithium-ion batteries. Adv. Mater 21(22), 2299 (2009).
17. H-Z. Geng, K.K. Kim, K.P. So, Y.S. Lee, Y. Chang, and Y.H. Lee: Effect of acid treatment on carbon nanotube-based flexible transparent conducting films. J. Am. Chem. Soc. 129(25), 7758 (2007).

18. J.W. Jo, J.W. Jung, J.U. Lee, and W.H. Jo: Fabrication of highly conductive and transparent thin films from single-walled carbon nanotubes using a new non-ionic surfactant via spin coating. ACS Nano 4(9), 5382 (2010).

19. S.L. Hellstrom, H.W. Lee, and Z. Bao: Polymer-assisted direct deposition of uniform carbon nanotube bundle networks for high performance transparent electrodes. ACS Nano 3(6), 1423 (2009).

20. Y. Hou, Y. Cheng, T. Hobson, and J. Liu: Design and synthesis of hierarchical $\mathrm{MnO}_{2}$ nanospheres/carbon nanotubes/conducting polymer ternary composite for high performance electrochemical electrodes. Nano Lett. 10(7), 2727 (2010)

21. X. Jia, C. Yan, Z. Chen, R. Wang, Q. Zhang, L. Guo, F. Wei, and Y. Lu: Direct growth of flexible $\mathrm{LiMn}_{2} \mathrm{O}_{4} / \mathrm{CNT}$ lithium-ion cathodes. Chem. Commun. 47(34), 9669 (2011).

22. J. Cheng, B. Wang, H.L. Xin, C. Kim, F. Nie, X. Li, G. Yang, and $\mathrm{H}$. Huang: Conformal coating of $\mathrm{TiO}_{2}$ nanorods on a 3-D CNT scaffold by using a CNT film as a nanoreactor: A freestanding and binder-free Li-ion anode. J. Mater. Chem. A 2(8), 2701 (2014).

23. G. Zhou, F. Li, and H-M. Cheng: Progress in flexible lithium batteries and future prospects. Energy Environ. Sci. 7(4), 1307 (2014).

24. K. Fu, O. Yildiz, H. Bhanushali, Y. Wang, K. Stano, L. Xue, X. Zhang, and P.D. Bradford: Aligned carbon nanotube-silicon sheets: A novel nano-architecture for flexible lithium ion battery electrodes. Adv. Mater. 25(36), 5109 (2013).

25. Z. Chen, J.W. To, C. Wang, Z. Lu, N. Liu, A. Chortos, L. Pan, F. Wei, Y. Cui, and Z. Bao: A three-dimensionally interconnected carbon nanotube-conducting polymer hydrogel network for high-performance flexible battery electrodes. Adv. Energy. Mater. 4(12), 1400507 (2014).

26. A.B. Dalton, S. Collins, E. Muñoz, J.M. Razal, V.H. Ebron, J.P. Ferraris, J.N. Coleman, B.G. Kim, and R.H. Baughman: Super-tough carbon-nanotube fibres. Nature 423(6941), 703 (2003).

27. Y.L. Li, I.A. Kinloch, and A.H. Windle: Direct spinning of carbon nanotube fibers from chemical vapor deposition synthesis. Science 304(5668), 276 (2004).

28. H. Lin, W. Weng, J. Ren, L. Qiu, Z. Zhang, P. Chen, X. Chen, J. Deng, Y. Wang, and H. Peng: Twisted aligned carbon nanotube/silicon composite fiber anode for flexible wire-shaped lithium-ion battery. Adv. Mater. 26(8), 1217 (2014).

29. K. Novoselov and A. Geim: The rise of graphene. Nat. Mater 6, 183 (2007)

30. S. Park and R.S. Ruoff: Chemical methods for the production of graphenes. Nat. Nanotechnol. 4(4), 217 (2009).

31. D. Wang, R. Kou, D. Choi, Z. Yang, Z. Nie, J. Li, L.V. Saraf, D. Hu, J. Zhang, and G.L. Graff: Ternary self-assembly of ordered metal oxide-graphene nanocomposites for electrochemical energy storage. ACS Nano 4(3), 1587 (2010).

32. K.S. Novoselov, A.K. Geim, S. Morozov, D. Jiang, Y. Zhang, S.A. Dubonos, I. Grigorieva, and A. Firsov: Electric field effect in atomically thin carbon films. Science 306(5696), 666 (2004).

33. K.S. Kim, Y. Zhao, H. Jang, S.Y. Lee, J.M. Kim, K.S. Kim, J-H. Ahn, P. Kim, J-Y. Choi, and B.H. Hong: Large-scale pattern growth of graphene films for stretchable transparent electrodes. Nature 457(7230), 706 (2009)

34. A. Reina, X. Jia, J. Ho, D. Nezich, H. Son, V. Bulovic, M.S. Dresselhaus, and J. Kong: Large area, few-layer graphene 
films on arbitrary substrates by chemical vapor deposition. Nano Lett. 9(1), 30 (2008).

35. C. Mattevi, H. Kim, and M. Chhowalla: A review of chemical vapour deposition of graphene on copper. J. Mater. Chem. 21(10), 3324 (2011).

36. S. Stankovich, D.A. Dikin, R.D. Piner, K.A. Kohlhaas, A. Kleinhammes, Y. Jia, Y. Wu, S.T. Nguyen, and R.S. Ruoff: Synthesis of graphene-based nanosheets via chemical reduction of exfoliated graphite oxide. Carbon 45(7), 1558 (2007).

37. Y. Hernandez, V. Nicolosi, M. Lotya, F.M. Blighe, Z. Sun, S. De, I. McGovern, B. Holland, M. Byrne, and Y.K. Gun'Ko: High-yield production of graphene by liquid-phase exfoliation of graphite. Nat. Nanotechnol. 3(9), 563 (2008).

38. R. Raccichini, A. Varzi, S. Passerini, and B. Scrosati: The role of graphene for electrochemical energy storage. Nat. Mater. 14(3), 271 (2015).

39. C. Wang, D. Li, C.O. Too, and G.G. Wallace: Electrochemical properties of graphene paper electrodes used in lithium batteries. Chem. Mater. 21(13), 2604 (2009).

40. A. Pandolfo and A. Hollenkamp: Carbon properties and their role in supercapacitors. J. Power Sources 157(1), 11 (2006).

41. D. Li, M.B. Muller, S. Gilje, R.B. Kaner, and G.G. Wallace: Processable aqueous dispersions of graphene nanosheets. Nat. Nanotechnol. 3(2), 101 (2008).

42. H. Chen, M.B. Müller, K.J. Gilmore, G.G. Wallace, and D. Li: Mechanically strong, electrically conductive, and biocompatible graphene paper. Adv. Mater. 20(18), 3557 (2008).

43. Q. Wu, Y. Xu, Z. Yao, A. Liu, and G. Shi: Supercapacitors based on flexible graphene/polyaniline nanofiber composite films. ACS Nano 4(4), 1963 (2010).

44. X. Yang, J. Zhu, L. Qiu, and D. Li: Bioinspired effective prevention of restacking in multilayered graphene films: Towards the next generation of high-performance supercapacitors. Adv. Mater. 23(25), 2833 (2011).

45. B. Shen, W. Zhai, and W. Zheng: Ultrathin flexible graphene film: An excellent thermal conducting material with efficient EMI shielding. Adv. Funct. Mater. 24(28), 4542 (2014).

46. Y. Gao, L-Q. Liu, S-Z. Zu, K. Peng, D. Zhou, B-H. Han, and Z. Zhang: The effect of interlayer adhesion on the mechanical behaviors of macroscopic graphene oxide papers. ACS Nano 5(3), 2134 (2011).

47. Q. Wang, J.L. Mynar, M. Yoshida, E. Lee, M. Lee, K. Okuro, K. Kinbara, and T. Aida: High-water-content mouldable hydrogels by mixing clay and a dendritic molecular binder. Nature 463(7279), 339 (2010).

48. Y. Tian, Y. Cao, Y. Wang, W. Yang, and J. Feng: Realizing ultrahigh modulus and high strength of macroscopic graphene oxide papers through crosslinking of mussel-inspired polymers. Adv. Mater. 25(21), 2980 (2013).

49. M.J. LaVoie, B.L. Ostaszewski, A. Weihofen, M.G. Schlossmacher, and D.J. Selkoe: Dopamine covalently modifies and functionally inactivates parkin. Nat. Med. 11(11), 1214 (2005).

50. Z. Chen, W. Ren, L. Gao, B. Liu, S. Pei, and H-M. Cheng: Three-dimensional flexible and conductive interconnected graphene networks grown by chemical vapour deposition. Nat. Mater. 10(6), 424 (2011).

51. T.H. Han, W.J. Lee, D.H. Lee, J.E. Kim, E.Y. Choi, and S.O. Kim: Peptide/graphene hybrid assembly into core/shell nanowires. Adv. Mater. 22(18), 2060 (2010).

52. X. Zhao, C.M. Hayner, M.C. Kung, and H.H. Kung: Flexible holey graphene paper electrodes with enhanced rate capability for energy storage applications. ACS Nano 5(11), 8739 (2011).

53. Z. Chen, W. Ren, L. Gao, B. Liu, S. Pei, and H.M. Cheng: Three-dimensional flexible and conductive interconnected graphene networks grown by chemical vapour deposition. Nat. Mater. 10(6), 424 (2011).

54. K. Xi, P.R. Kidambi, R. Chen, C. Gao, X. Peng, C. Ducati, S. Hofmann, and R.V. Kumar: Binder free three-dimensional sulphur/few-layer graphene foam cathode with enhanced high-rate capability for rechargeable lithium sulphur batteries. Nanoscale 6(11), 5746 (2014).

55. X. Huang, K. Qian, J. Yang, J. Zhang, L. Li, C. Yu, and D. Zhao: Functional nanoporous graphene foams with controlled pore sizes. Adv. Mater. 24(32), 4419 (2012).

56. F. Liu, S. Song, D. Xue, and H. Zhang: Folded structured graphene paper for high performance electrode materials. $A d v$. Mater. 24(8), 1089 (2012).

57. A. Yu, I. Roes, A. Davies, and Z. Chen: Ultrathin, transparent, and flexible graphene films for supercapacitor application. Appl. Phys. Lett. 96(25), 253105 (2010).

58. H. Gwon, H-S. Kim, K.U. Lee, D-H. Seo, Y.C. Park, Y-S. Lee, B.T. Ahn, and K. Kang: Flexible energy storage devices based on graphene paper. Energy Environ. Sci. 4(4), 1277 (2011).

59. A. Yu, H.W. Park, A. Davies, D.C. Higgins, Z. Chen, and X. Xiao: Free-Standing layer-by-layer hybrid thin film of graphene- $\mathrm{MnO}_{2}$ nanotube as anode for lithium ion batteries. J. Phys. Chem. Lett. 2(15), 1855 (2011).

60. D. Deng, M.G. Kim, J.Y. Lee, and J. Cho: Green energy storage materials: Nanostructured $\mathrm{TiO}_{2}$ and $\mathrm{Sn}$-based anodes for lithiumion batteries. Energy Environ Sci. 2(8), 818 (2009).

61. B. Wang, X. Li, X. Zhang, B. Luo, M. Jin, M. Liang, S.A. Dayeh, S. Picraux, and L. Zhi: Adaptable silicon-carbon nanocables sandwiched between reduced graphene oxide sheets as lithium ion battery anodes. ACS Nano 7(2), 1437 (2013).

62. X. Huang, B. Sun, K. Li, S. Chen, and G. Wang: Mesoporous graphene paper immobilised sulfur as a flexible electrode for lithium-sulfur batteries. J. Mater. Chem. A 1(43), 13484 (2013).

63. W-M. Zhang, X-L. Wu, J-S. Hu, Y-G. Guo, and L-J. Wan: Carbon coated $\mathrm{Fe}_{3} \mathrm{O}_{4}$ nanospindles as a superior anode material for lithium-ion batteries. Adv. Funct. Mater. 18(24), 3941 (2008).

64. R. Wang, C. Xu, J. Sun, L. Gao, and C. Lin: Flexible freestanding hollow $\mathrm{Fe}_{3} \mathrm{O}_{4}$ /graphene hybrid films for lithium-ion batteries. J. Mater. Chem. A 1(5), 1794 (2013).

65. R. Wang, C. Xu, J. Sun, Y. Liu, L. Gao, and C. Lin: Free-standing and binder-free lithium-ion electrodes based on robust layered assembly of graphene and $\mathrm{Co}_{3} \mathrm{O}_{4}$ nanosheets. Nanoscale 5(15), 6960 (2013).

66. H. Ji, L. Zhang, M.T. Pettes, H. Li, S. Chen, L. Shi, R. Piner, and R.S. Ruoff: Ultrathin graphite foam: A three-dimensional conductive network for battery electrodes. Nano Lett. 12(5), 2446 (2012).

67. X. Li, T. Zhao, K. Wang, Y. Yang, J. Wei, F. Kang, D. Wu, and H. Zhu: Directly drawing self-assembled, porous, and monolithic graphene fiber from chemical vapor deposition grown graphene film and its electrochemical properties. Langmuir 27(19), 12164 (2011).

68. E.Y. Jang, J. Carretero-González, A. Choi, W.J. Kim, M.E. Kozlov, T. Kim, T.J. Kang, S.J. Baek, D.W. Kim, and Y.W. Park: Fibers of reduced graphene oxide nanoribbons. Nanotechnology 23(23), 235601 (2012).

69. Z. Tian, C. Xu, J. Li, G. Zhu, Z. Shi, and Y. Lin: Self-assembled free-standing graphene oxide fibers. ACS Appl. Mater. Interfaces 5(4), 1489 (2013).

70. Z. Xu and C. Gao: Graphene chiral liquid crystals and macroscopic assembled fibres. Nat. Commun. 2, 571 (2011).

71. Z. Xu, H. Sun, X. Zhao, and C. Gao: Ultrastrong fibers assembled from giant graphene oxide sheets. Adv. Mater. 25(2), 188 (2013).

72. Y. Zhao, C. Jiang, C. Hu, Z. Dong, J. Xue, Y. Meng, N. Zheng, P. Chen, and L. Qu: Large-scale spinning assembly of neat, 
morphology-defined, graphene-based hollow fibers. ACS Nano 7(3), 2406 (2013).

73. S.H. Aboutalebi, R. Jalili, D. Esrafilzadeh, M. Salari, Z. Gholamvand, S. Aminorroaya Yamini, K. Konstantinov, R.L. Shepherd, J. Chen, and S.E. Moulton: High-performance multifunctional graphene yarns: Toward wearable all-carbon energy storage textiles. ACS Nano 8(3), 2456 (2014).

74. C. Xiang, C.C. Young, X. Wang, Z. Yan, C.C. Hwang, G. Cerioti, J. Lin, J. Kono, M. Pasquali, and J.M. Tour: Large flake graphene oxide fibers with unconventional $100 \%$ knot efficiency and highly aligned small flake graphene oxide fibers. Adv. Mater. 25(33), 4592 (2013).

75. Z. Dong, C. Jiang, H. Cheng, Y. Zhao, G. Shi, L. Jiang, and L. Qu: Facile fabrication of light, flexible and multifunctional graphene fibers. Adv. Mater. 24(14), 1856 (2012).

76. Q. Chen, Y. Meng, C. Hu, Y. Zhao, H. Shao, N. Chen, and L. Qu: $\mathrm{MnO}_{2}$-modified hierarchical graphene fiber electrochemical supercapacitor. J. Power Sources 247, 32 (2014).

77. Y. Hu, X. Li, J. Wang, R. Li, and X. Sun: Free-standing graphene-carbon nanotube hybrid papers used as current collector and binder free anodes for lithium ion batteries. J. Power Sources 237, 41 (2013).

78. L. Qiu, X. Yang, X. Gou, W. Yang, Z.F. Ma, G.G. Wallace, and D. Li: Dispersing carbon nanotubes with graphene oxide in water and synergistic effects between graphene derivatives. Chem. Eur. J. 16(35), 10653 (2010).

79. H. Sun, X. You, J. Deng, X. Chen, Z. Yang, J. Ren, and H. Peng: Novel graphene/carbon nanotube composite fibers for efficient wire-shaped miniature energy devices. Adv. Mater. 26(18), 2868 (2014).

80. L. Kou, T. Huang, B. Zheng, Y. Han, X. Zhao, K. Gopalsamy, H. Sun, and C. Gao: Coaxial wet-spun yarn supercapacitors for high-energy density and safe wearable electronics. Nat. Commun. 5, 536 (2014).

81. G. Wang, H. Wang, X. Lu, Y. Ling, M. Yu, T. Zhai, Y. Tong, and Y. Li: Solid-state supercapacitor based on activated carbon cloths exhibits excellent rate capability. Adv. Mater. 26(17), 2676 (2014).

82. H. Yu, C. Zhu, K. Zhang, Y. Chen, C. Li, P. Gao, P. Yang, and Q. Ouyang: Three-dimensional hierarchical $\mathrm{MoS}_{2}$ nanoflake array/carbon cloth as high-performance flexible lithium-ion battery anodes. J. Mater. Chem. A 2(13), 4551 (2014).

83. B. Liu, J. Zhang, X. Wang, G. Chen, D. Chen, C. Zhou, and G. Shen: Hierarchical three-dimensional $\mathrm{ZnCo}_{2} \mathrm{O}_{4}$ nanowire arrays/carbon cloth anodes for a novel class of highperformance flexible lithium-ion batteries. Nano Lett. 12(6), 3005 (2012).

84. Y. Sun, R.B. Sills, X. Hu, Z.W. Seh, X. Xiao, H. Xu, W. Luo, H. Jin, Y. Xin, T. Li, Z. Zhang, J. Zhou, W. Cai, Y. Huang, and Y. Cui: A bamboo-inspired nanostructure design for flexible, foldable, and twistable energy storage devices. Nano Lett. 15(6), 3899 (2015).

85. H. Wu, Z. Lou, H. Yang, and G. Shen: A flexible spiral-type supercapacitor based on $\mathrm{ZnCo}_{2} \mathrm{O}_{4}$ nanorod electrodes. Nanoscale 7(5), 1921 (2015).

86. G-W. Yang, C-L. Xu, and H-L. Li: Electrodeposited nickel hydroxide on nickel foam with ultrahigh capacitance. Chem. Commun. 48, 6537 (2008).
87. Y. Liu, K. Huang, Y. Fan, Q. Zhang, F. Sun, T. Gao, L. Yang, and J. Zhong: Three-dimensional network current collectors supported $\mathrm{Si}$ nanowires for lithium-ion battery applications. Electrochim. Acta 88, 766 (2013).

88. Q. Zhao, X. Hu, K. Zhang, N. Zhang, Y. Hu, and J. Chen: Sulfur nanodots electrodeposited on $\mathrm{Ni}$ foam as high-performance cathode for Li-S batteries. Nano Lett. 15(1), 721 (2015).

89. X. Li, J. Yang, Y. Hu, J. Wang, Y. Li, M. Cai, R. Li, and X. Sun: Novel approach toward a binder-free and current collector-free anode configuration: Highly flexible nanoporous carbon nanotube electrodes with strong mechanical strength harvesting improved lithium storage. J. Mater. Chem. 22(36), 18847 (2012).

90. Y. Won, A. Kim, W. Yang, S. Jeong, and J. Moon: A highly stretchable, helical copper nanowire conductor exhibiting a stretchability of $700 \%$. NPG Asia Mater 6(9), e132 (2014).

91. S. Jiang, H. Zhang, S. Song, Y. Ma, J. Li, G.H. Lee, Q. Han, and J. Liu: Highly stretchable conductive fibers from few-walled carbon nanotubes coated on poly(m-phenylene isophthalamide) Polymer core/shell structures. ACS Nano 9(10), 10252 (2015).

92. K. Jost, C.R. Perez, J.K. McDonough, V. Presser, M. Heon, G. Dion, and Y. Gogotsi: Carbon coated textiles for flexible energy storage. Energy Environ. Sci. 4(12), 5060 (2011).

93. G. Zhou, S. Pei, L. Li, D.W. Wang, S. Wang, K. Huang, L.C. Yin, F. Li, and H.M. Cheng: A graphene-pure-sulfur sandwich structure for ultrafast, long-life lithium-sulfur batteries. Adv. Mater. 26(4), 625 (2014).

94. G. Zhou, L. Li, C. Ma, S. Wang, Y. Shi, N. Koratkar, W. Ren, F. Li, and H-M. Cheng: A graphene foam electrode with high sulfur loading for flexible and high energy Li-S batteries. Nano Energy 11, 356 (2015).

95. T. Chen, H. Peng, M. Durstock, and L. Dai: High-performance transparent and stretchable all-solid supercapacitors based on highly aligned carbon nanotube sheets. Sci. Rep. 4, 204 (2014).

96. H. Lee, J-K. Yoo, J-H. Park, J.H. Kim, K. Kang, and Y.S. Jung: A stretchable polymer-carbon nanotube composite electrode for flexible lithium-ion batteries: Porosity engineering by controlled phase separation. Adv. Energy. Mater. 2(8), 976 (2012).

97. L. Hu, M. Pasta, F.L. Mantia, L. Cui, S. Jeong, H.D. Deshazer, J.W. Choi, S.M. Han, and Y. Cui: Stretchable, porous, and conductive energy textiles. Nano Lett. 10(2), 708 (2010).

98. M. Koo, K.I. Park, S.H. Lee, M. Suh, D.Y. Jeon, J.W. Choi, K. Kang, and K.J. Lee: Bendable inorganic thin-film battery for fully flexible electronic systems. Nano Lett. 12(9), 4810 (2012).

99. P. Barpanda, J.N. Chotard, C. Delacourt, M. Reynaud, Y. Filinchuk, M. Armand, M. Deschamps, and J.M. Tarascon: $\mathrm{LiZnSO}_{4} \mathrm{~F}$ made in an ionic liquid: A ceramic electrolyte composite for solid-state lithium batteries. Angew. Chem., Int. Ed. 50(11), 2526 (2011).

100. H-J. Ha, E-H. Kil, Y.H. Kwon, J.Y. Kim, C.K. Lee, and S-Y. Lee: UV-curable semi-interpenetrating polymer networkintegrated, highly bendable plastic crystal composite electrolytes for shape-conformable all-solid-state lithium ion batteries Energy Environ. Sci. 5(4), 6491 (2012).

101. E.H. Kil, K.H. Choi, H.J. Ha, S. Xu, J.A. Rogers, M.R. Kim, Y.G. Lee, K.M. Kim, K.Y. Cho, and S.Y. Lee: Imprintable, bendable, and shape-conformable polymer electrolytes for versatile-shaped lithium-ion batteries. Adv. Mater. 25(10), 1395 (2013). 